\title{
Reexamining the Binary Interaction of Four Pairs of Tropical Cyclones in the Northwest Pacific
}

\author{
Wei-hong QIAN, Jing HUANG, and Guang-wen ZHANG \\ Department of Atmospheric and Oceanic Sciences, Peking University, Beijing, China
}

(Manuscript received 13 June 2015, in final form 11 February 2016)

\begin{abstract}
Four pairs of tropical cyclones (TCs) in the vicinity of the Northwest (NW) Pacific have been restudied in this paper by decomposing a total flow into a climatic component and an anomaly as well as by a simple generalized beta-advection model (GBAM). These results contradict with previous reports that binary interactions occurred in the four pairs of TCs. The intensities of the TC pairs were not well matched to each other during their common lifetime period; thus, the result of GBAM revealed that the strong TC had a direct influence on the track of the weak TC, but the reverse was not true. All dynamical models for studying the binary interaction of two adjacent TCs depend on how to separate the TC vortex and surrounding flow from the total flow. In this paper, the anomalous component can be directly used to measure the intensities and sizes of TCs and other adjacent disturbances. The track of TC as an anomalous vortex is influenced by the climatic steering flow and interacts with other anomalous systems in the vicinity. Whether a binary interaction happens between two TCs and whether the two TCs interact with other anomalous systems can be determined by the GBAM at the optimal level near the maximum center of the vorticity anomaly.
\end{abstract}

Keywords binary interaction; tropical cyclone; simple model; Northwest Pacific

\section{Introduction}

Previous studies have shown that tropical cyclone (TC) motions are mainly governed by the tropospheric average steering flow (Chan and Gray 1982), which is generally defined as the vertical weighted average of the flow in the troposphere. During summer and autumn, TCs in the Northwest (NW) Pacific generally move westward, northwestward, or northward, mainly driven by large-scale steering flow associated with the dominant NW Pacific subtropical high and the transient trough-ridge sequences coming from the west in middle and high latitudes. After passing the ridge line of the NW Pacific subtropical

Corresponding author: Wei-hong Qian, Department of Atmospheric and Oceanic Sciences, Peking University, Beijing 100871, China

E-mail: qianwh@pku.edu.cn

(C)2016, Meteorological Society of Japan high, TCs accelerate northeastward owing to the influence of the large-scale westerly steering flow. This type of TC motion is considered as the normal track and designated as the recurvature (George and Gray 1977). There were, however, some unusual tracks that do not follow the large-scale steering flows, but turn left and/or right on one or several occasions. It is, thus, difficult to forecast whether, when, and where the turning motion occurs based on the traditional synoptic chart and the numerical weather forecast model products. In mainland China, forecasters are particularly concerned with three types of unusual TC tracks. One of them is the sudden rightturning track of TCs when they move westward from the NW Pacific to the South China Sea (SCS) (Deng et al. 2010). Another is the sudden left-turning (westturning) TCs that occur in the East China seas (Wu et al. 2013) when they move northward. The above two types of TCs can cause direct damage in China 
when they rapidly turn and make landfall in China. The third type of unusual TC tracks is the binary interaction of two nearby TCs in the NW Pacific. The short-term (2-3 days) track prediction of this type of TC is still difficult in practice (Wu et al. 2003; Yang et al. 2008; Wu et al. 2011; Wu et al. 2012; Xu et al. 2013; Bai et al. 2014).

Many previous studies have investigated these three types of unusual TCs in the NW Pacific and the SCS. It can be summarized that these unusual TC tracks are influenced by not only large-scale steering flows but also other nearby high and low circulation systems such as subtropical highs, vortices, and terrains (Chen and Ding 1979; Lu and Qian 2012; Jang and Chun 2012). Therefore, it is essential to separate the largescale steering flows and TC vortices from observation in order to understand the interaction mechanism. Recently, Qian et al. (2014) developed a generalized beta-advection model (GBAM) by decomposing an atmospheric variable into a climatic component and an anomaly. The large-scale steering flows are derived from the climatic component, and the anomaly can be used to reasonably identify TC vortices and other disturbances. Fifteen cases of sudden right-turning TCs in the SCS and four sudden left-turning TCs in the East China seas from 1979 to 2011, as unusual TC tracks, were studied using this decomposition. All 15 right-turning and 10 straight-moving TC tracks in the SCS as well as 99 right-turning, 30 straight-turning, and four left-turning TCs in the East China seas were successfully forecasted by the GBAM 2-3 days in advance (Qian et al. 2014; Huang et al. 2015).

Given its success in understanding and predicting unusual TC tracks in the SCS and East China seas, the GBAM is used to study the binary interaction of unusual TC tracks in the NW Pacific based on previous studies. As we have previously studied the TCs in the East China seas, four unusual left-turning TCs have been identified in the literature. In this paper, we will study four TC pairs in the East China seas, which have been documented in previous studies (Wu et al. 2003; Yang et al. 2008; Wu et al. 2012; Xu et al. 2013). Among them, two TC pairs were investigated by complex model experiments and another two were studied using potential vorticity diagnosis. Using our decomposition method and the GBAM, this paper is organized as follows. Section 2 describes the data and methods including four pairs of TCs, the variable decomposition method, and the simple GBAM. Thermodynamical structures and interactions of four TC pairs are reexamined using the decomposition method and the GBAM, respectively, in Section 3. Conclu- sions and discussion are given in Section 4.

\section{Data and method}

\subsection{Data and four pair TCs}

Two datasets are used in this paper. The first dataset is the ECMWF Re-Analysis Interim (ERA interim) product from the website (http://www.ecmwf.int/ research/era/do/get/index), with a horizontal $0.75^{\circ} \times$ $0.75^{\circ}$ longitude-latitude grid interval and 37 vertical levels (Dee et al. 2011). The second dataset is the best tracks derived from the Joint Typhoon Warning Center (Chu et al. 2002). Since the observations of the "modern satellite" era started in 1979, the dataset from 1979 to the present is used.

Since the four TC pairs have been studied in previous works, we only simply describe their tracks and intensities. The details for each TC can be found in the literature. In Figs. 1a and 1b, Saomai generated at 1800 UTC, 1 September 2000 in the tropical NW Pacific and slowly moved westward. After a local circle movement, it moved northwestward and its intensity (140 knots) peaked at 1200 UTC, 10 September 2000. Before it rapidly turned right in the East China Seas, its speed was very slow. Bopha occurred at 1800 UTC, 5 September 2000 in the heading direction of Saomai and reached its strongest (55 knots) at 1200 UTC, 10 September 2000 near the southeast of Taiwan Island. The lifetime period of Bopha was shorter and weaker than that of Saomai (Fig. 1b). Bopha experienced a left-turning or southward movement in the east of Taiwan Island, while Saomai followed a northwestward course.

The second pair of TCs was Fengshen (2002) and Fungwong (2002), shown in Figs. 1c and 1d. Fengshen generated at 0000 UTC, 14 July 2002 in the tropical NW Pacific and moved northwestward before finally dissipating in the Yellow Sea at 0600 UTC, 27 July 2002. Its intensity (145 knots) peaked at 1200 UTC, 21 July 2002. Fungwong occurred at 0000 UTC, 20 July 2002 in the NW of Fengshen and reached its strongest (65 knots) at $0600 \mathrm{UTC}, 23$ July 2002. The lifetime period of Fungwong was shorter and weaker than that of Fengshen. After experiencing a left-turning circle movement, Fungwong dissipated in the Yellow Sea at 1800 UTC, 27 July 2002.

In Figs. 1e and 1f, the two TCs, Saomai and Bopha, both moved northwestward after generating at 0000 and 0000 UTC, 4 and 5 August 2006, respectively, in the tropical NW Pacific. Their intensities (140 and 55 knots) peaked at 1200 and 1800 UTC, 9 and 8 August 2006, respectively, when they were getting closer. Finally, Bopha dissipated near the South China coast 
(a) 2000(Bopha \& Saomai)

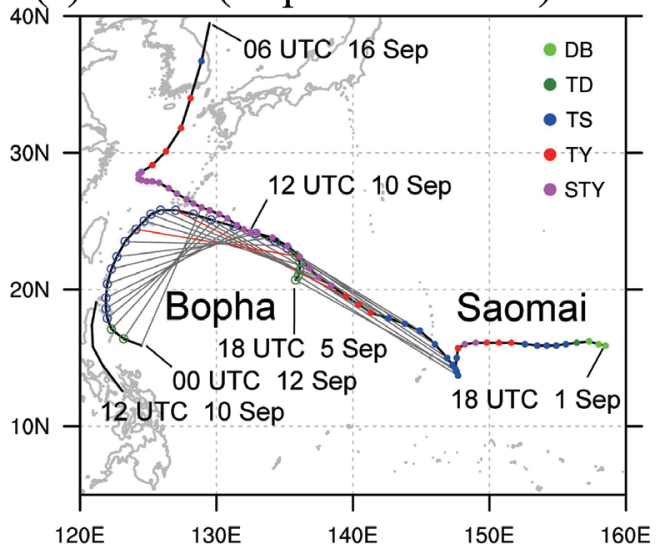

(b)

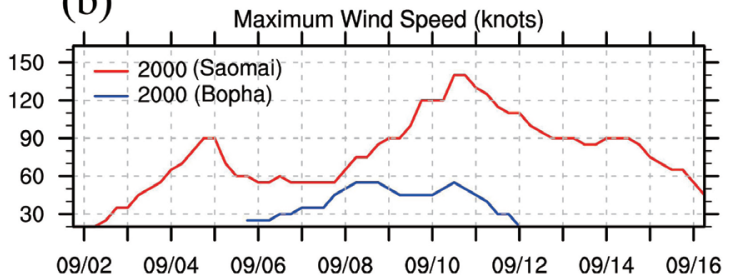

(e) 2006(Bopha \& Saomai)

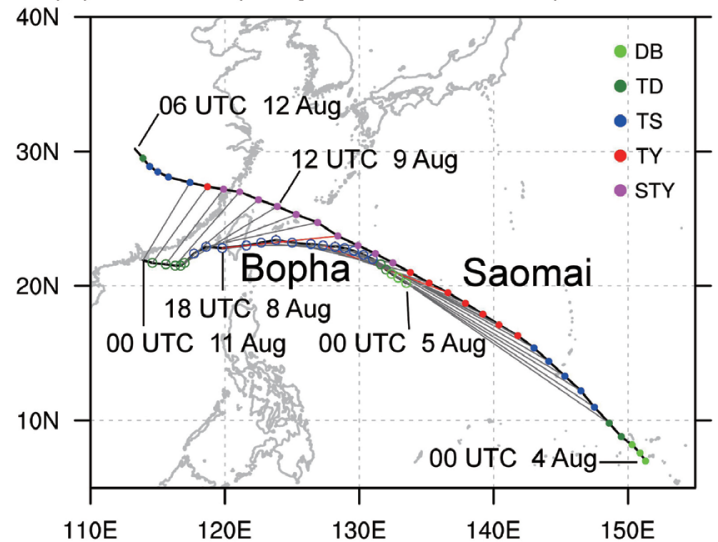

(f)

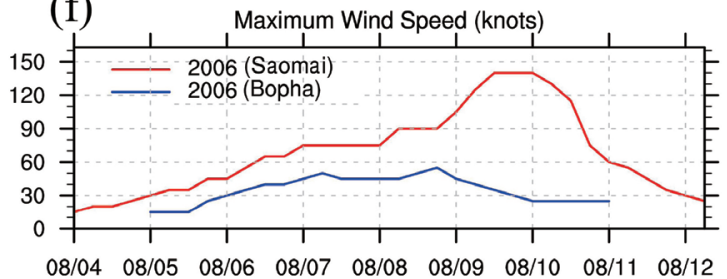

(c) 2002(Fengshen \& Fungwong)

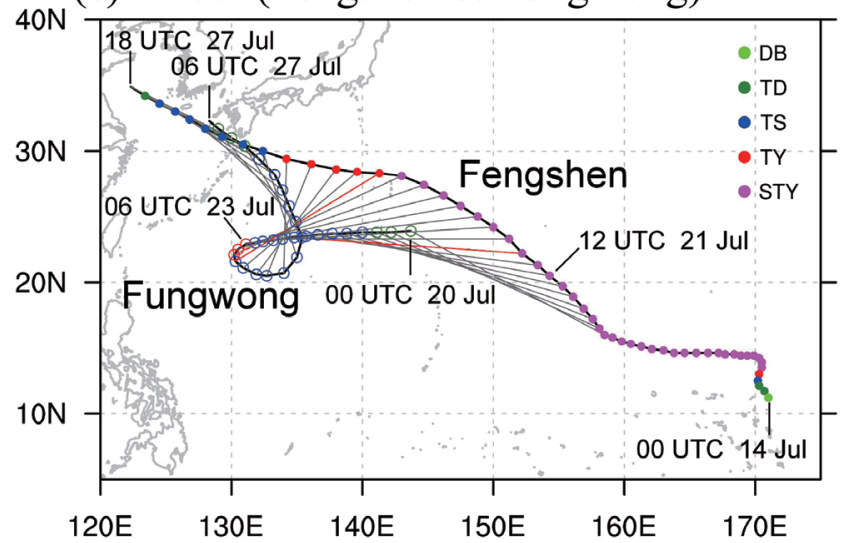

(d)

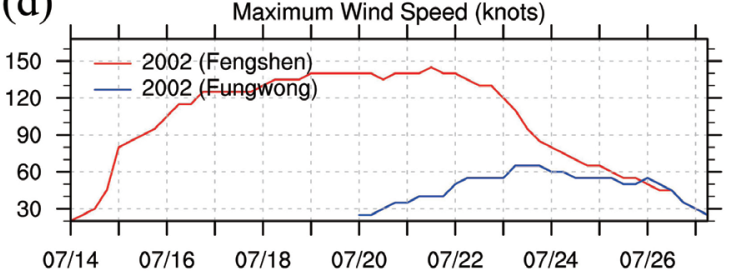

(g)

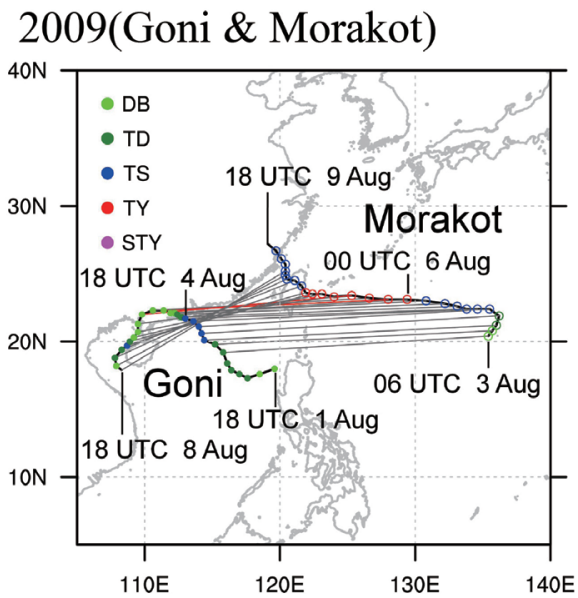

(h)

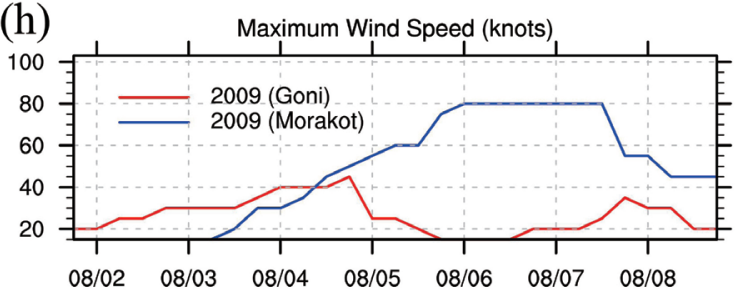

Fig. 1. Tracks and intensities of four TC pairs: (a, b) Bopha and Saomai (2000), (c, d) Fengshen and Fungwong (2002), (e, f) Bopha and Saomai (2006), and (g, h) Goni and Morakot (2009). Two TC tracks are drawn by hollow and solid dots respectively. The dot color indicates the TC scale: tropical disturbance (DB), tropical depression (TD), tropical storm (TS), typhoon (TY), and super typhoon (STY). Maximum surface wind speeds (knots) of two TCs at 6-h intervals are denoted by red and blue lines respectively in (b), (d), (f), and (h). The gray line in (a), (c), (e), and (g) connects a TC pair at the same time point while red lines connect two points used in Figs. 2, 7, 11, and 13. 
and Saomai dissipated in central China. The lifetime period of Bopha was shorter and its strength was weaker than that of Saomai in 2006 (Fig. 1f).

In recent years, the notorious and deadly $\mathrm{TC}$ Morakot (2009) hit Taiwan Island and caused severe damage (Wu et al. 2011; Wu et al. 2012; Lee et al. 2013; Liou et al. 2013; Yu and Cheng 2013; Tsai et al. 2013; Zheng et al. 2014; Chien 2014; Huang et al. 2014). Morakot generated at 0600 UTC, 3 August 2009 at $1600 \mathrm{~km}$ east of Taiwan Island and moved westward before making landfall on Taiwan Island at 1545 UTC, 8 August 2009 (Fig. 1g). Its intensity (80 knots) peaked at 0000 UTC, 6 August 2009. Goni (2009) generated in the northeastern SCS at 1800 UTC, 1 August 2009 before Morakot's formation. Goni experienced a slow movement in the northern SCS and the South China coast for 6 days with a very weak intensity and two intensity peaks (Fig. 1h). We are interested in the right-turning movement of Morakot after making landfall on Taiwan Island and examine its interaction with Goni.

\subsection{Decomposition Methodology}

Traditionally, a TC vortex is considered to be embedded in and steered by a large-scale environmental flow (Chen and Ding 1979; Chan and Gray 1982; Deng et al. 2010; Roy and Kovordanyi 2012). In all synoptic analyses and model experimental predictions, we need to know the TC vortex's size and intensity. Theoretically, this steering flow can be obtained by removing the TC vortex from the total circulation or total flow on the spatial scale of a thousand kilometers. However, since an actual circulation is complex and contributed by both the TC vortex (internal forcing) and surrounding flow (external forcing) as well as their interactions, it is difficult to define a TC vortex. Therefore, extracting a TC vortex from its surrounding flow remains a challenge.

The relationship between TC movement and largescale environmental flow usually varies depending on the definition of environmental flow. Previous works attempted to extract a steering flow from the total flow by spatially filtering the TC vortex and other smallscale disturbances. George and Gray (1976) suggested that the flow near $700 \mathrm{hPa}$ or the averaged flow in the $700-500 \mathrm{hPa}$ layer is the best representation of the environmental steering flow in the tropics, while Holland (1984) suggested the use of the vertical mean steering flow between 850 and $300 \mathrm{hPa}$. Some others suggested the use of a deep layer mean from 1000 to 150 or $100 \mathrm{hPa}$ to approximate a TC's vortex (Sanders et al. 1980; Dong and Neumann 1986; Franklin
1990; Velden and Leslie 1991). Recently, Galarneau and Davis (2013) defined an optimal environmental steering flow with variable vertical extent. At all levels from 850 to $200 \mathrm{hPa}$, at an increment of $50 \mathrm{hPa}$, a TC's vorticity and divergence are removed within a predetermined radius to obtain the environmental steering flow for a TC (Davis et al. 2008; Galarneau and Davis 2013). However, there is a large uncertainty in defining the radius of a TC vortex in this method. In short, it is difficult to obtain a realistic steering flow and TC vortex from all the existing methods.

A new objective approach is used to separate large-scale background flow and synoptic-scale disturbances in this study. The new approach can avoid the ambiguity in defining steering flow, TC vortex, and surrounding small-scale disturbances. This method decomposes a total meteorological field into a climatic component (an hour interval) and an anomaly. For any atmospheric field $F_{\{d, y\}}(\lambda, \varphi, p, t)$, such as geopotential height, temperature, and wind at diurnal time $t$ ( $24 \mathrm{~h}$ a day) on calendar date $d$ in year $y$ at a spatial point of longitude $\lambda$, latitude $\varphi$, and pressure level $p$ can be decomposed into a climatic field $\tilde{F}_{d}(\lambda, \varphi, p, t)$ and an anomaly field $F_{\{d, y\}}^{\prime}(\lambda, \varphi, p, t)$ following Qian (2012a, b), Qian et al. (2014), and Huang et al. (2015):

$$
F_{\{d, y\}}(\lambda, \varphi, p, t)=\tilde{F}_{d}(\lambda, \varphi, p, t)+F_{\{d, y\}}^{\prime}(\lambda, \varphi, p, t) .
$$

The climatic field is estimated by averaging over 30 years (1981-2010) based on the reanalysis data on a calendar date $d$ and at a diurnal time $t$,

$$
\tilde{F}_{d}(\lambda, \varphi, p, t)=\sum_{y=1981}^{2010} F_{\{d, y\}}(\lambda, \varphi, p, t) / 30,
$$

where $y$ covers 1981 to 2010 . We assumed that the positive and negative anomalies of atmospheric variables at a specific grid point and a given calendar time cancel each other out during the 30 year period to approximate the quasi-static climatic state. The climatology defined by Eq. (2) varies from hour to hour and from day to day.

This approach has been used in the diagnoses of weather extremes such as freezing rain (Qian and Zhang 2012), heat wave (Ding and Qian 2012), and regional heavy rains based on reanalysis datasets (Qian et al. 2012) as well as in the application of model outputs (Qian et al. 2013). This approach has also been used in complex and simple models (Peng et al. 2013; Qian et al. 2014; Huang et al. 2015). 


\subsection{Model descriptions}

A barotropic vorticity equation of horizontal winds without considering divergence, for frictionless and adiabatic atmospheric motion, can be simply written as in the planar coordinate system:

$$
\frac{\partial \varsigma}{\partial t}=-u \frac{\partial \varsigma}{\partial x}-v \frac{\partial \varsigma}{\partial y}-\beta v,
$$

where, $\beta=(d f / d y)=2 \Omega \cos \varphi / a, f=2 \Omega \sin \varphi$, and $\Omega=7.292 \times 10^{-5} \mathrm{rad} \mathrm{s}^{-1}$, which is the angular speed of Earth's rotation. $a$ and $\varphi$ are the mean radius of the Earth and geographical latitude, respectively. Eq. (3) indicates that the movement of a vortex such as a TC is primarily controlled by the advection of relative vorticity and the Earth rotation beta effect. This is the classical two-dimensional BAM.

Holland (1983) and Marks (1992) applied this BAM to hurricane track forecasting. The dynamics of advection and the beta effect of TC motion have been described in more detail by Chan (2005). There are several versions of BAM (Velden and Leslie 1991; Marks 1992; Simpson et al. 2003): the BAM shallow applies to the $850-700 \mathrm{hPa}$ layer, the BAM medium to the $850-400 \mathrm{hPa}$ layer, and the BAM deep to the 850-200 hPa layer. Establishing which layer (shallow, medium, or deep) to apply is an issue for the classical BAM. Note that this simple BAM is written at the non-divergence level. In our previous study with a GBAM (Qian et al. 2014), we found that there is an optimal level for a BAM-type model to apply for short-term (2-3 days) TC track predictions. This level is close to the levels of the maximum vorticity anomaly (max-VA) and minimum divergence anomaly (min-DA). The max-VA and min-DA levels can be estimated between 850 and $200 \mathrm{hPa}$ over the TC center at model initialization time.

After a detailed description in our recent work (Huang et al. 2015), a GBAM can be represented by the equation:

$$
\frac{\partial \varsigma^{\prime}}{\partial t}=-u \frac{\partial \varsigma^{\prime}}{\partial x}-v \frac{\partial \varsigma^{\prime}}{\partial y}-\beta v^{\prime}
$$

The GBAM basically describes the advection of a TC disturbance (anomaly) by the total flow, which is the sum of the climatic and anomalous flows (Eq. 1 ), while the beta effect is caused by the anomalous meridional flow. Eq.(4) is written in planar coordinates for easy understanding and simplicity. In our actual calculations, it is written and calculated in spherical coordinates.

$$
\frac{\partial \varsigma^{\prime}}{\partial t}=-\frac{u}{a \cos (\theta)} \frac{\partial \varsigma^{\prime}}{\partial \lambda}-\frac{v}{a} \frac{\partial \varsigma^{\prime}}{\partial \theta}-\beta v^{\prime},
$$

where the vorticity anomaly $\varsigma^{\prime}=\frac{1}{a \cos (\theta)} \frac{\partial v^{\prime}}{\partial \lambda}-\frac{1}{a} \frac{\partial u^{\prime}}{\partial \theta}$.

We found that neither the climatic nor the anomalous flows alone can explain unusual tracks. Many TC experiments carried out by Huang et al. (2015) showed that the ability of the GBAM to predict tracks 1-2 days in advance apparently outperformed the classical BAMs for both the left- and right-turning TC tracks. In this paper, we do not compare the ability among the GBAM and classical BAMs. Instead, we directly apply the GBAM to examine the binary interaction of two TCs or the interaction between a TC and another anomalous system in the vicinity.

A brief description of the computational methods used in the GBAM is introduced in the following. For details readers can refer to Qian et al. (2014) and Huang et al. (2015). The GBAM integration is done in spherical coordinates with a horizontal $0.75^{\circ} \times$ $0.75^{\circ}$ longitude-latitude grid spacing at the optimal pressure level. The ERA interim product is used as the initial conditions, where the global climate and anomaly are obtained through Eqs. (1) and (2). The time step for the model integration is $10 \mathrm{~min}$, and the climate is updated every $6 \mathrm{~h}$ (since the reanalysis is available every $6 \mathrm{~h}$ ) through Eq. (2). At each time step, a new vorticity anomaly is calculated using Eq. (5). Then, a new wind anomaly is derived from the new vorticity anomaly via a spherical harmonic expansion, and climatic wind is linearly interpolated between two climatic winds that are $6 \mathrm{~h}$ apart (ideally climatic winds are needed at every time step, i.e., 10 $\mathrm{min})$. Since the vorticity anomaly tends to grow too strong and causes numerical instability during integration, the model-derived new vorticity anomalies are smoothed by performing a triangular truncation on the spherical harmonic coefficients to make the model computationally stable. This technique stabilizes the computation but also produces a side effect; the amplitude of vorticity anomaly decreases steadily with time and becomes weaker than the observations after 2 days. The spherical harmonic expansion and triangular truncation technique are described on this website: http://dx.doi.org/10.5065/D6WD3XH5.

\section{Structures and interactions}

\subsection{Bopha and Saomai (2000)}

Saomai experienced a long-lived track, while Bopha appeared only from 1800 UTC, 5 September 2000 to 0000 UTC, 12 September 2000 (Figs. 1a, b). 
(a)

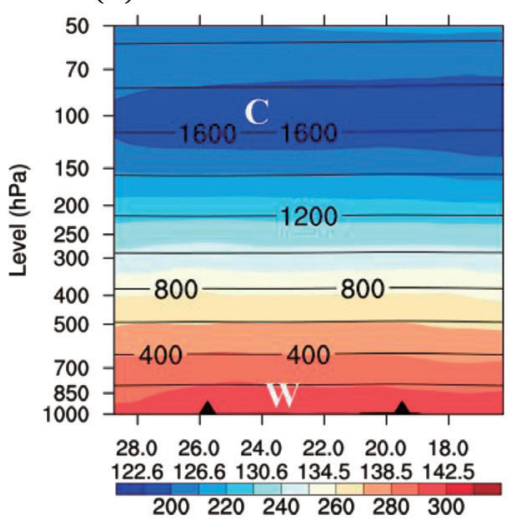

(d)

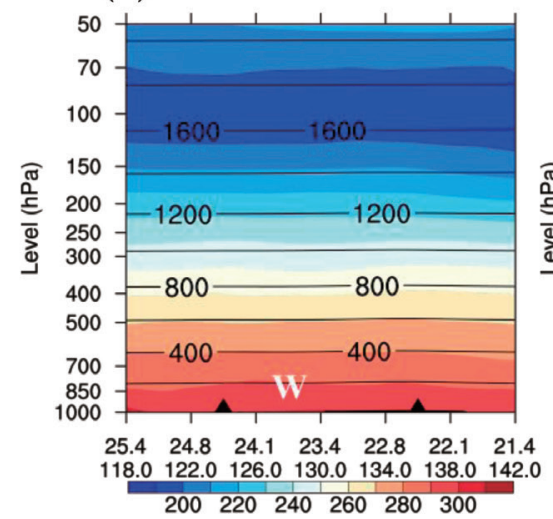

(b)

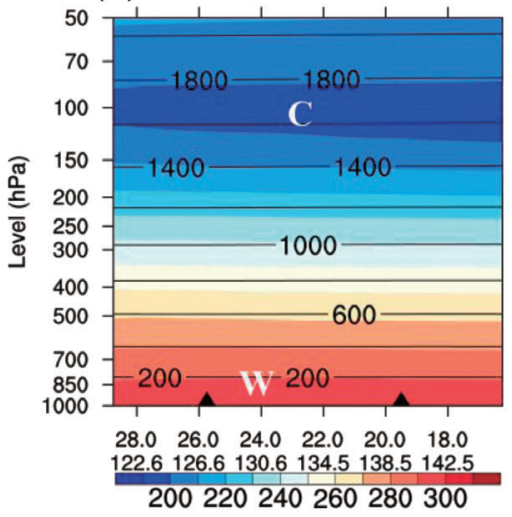

(e)

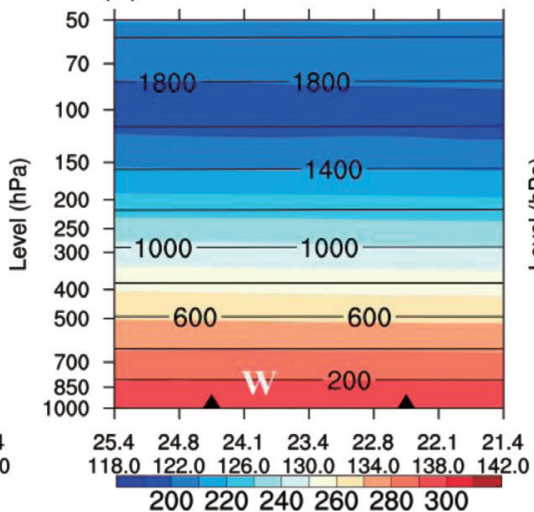

(c)

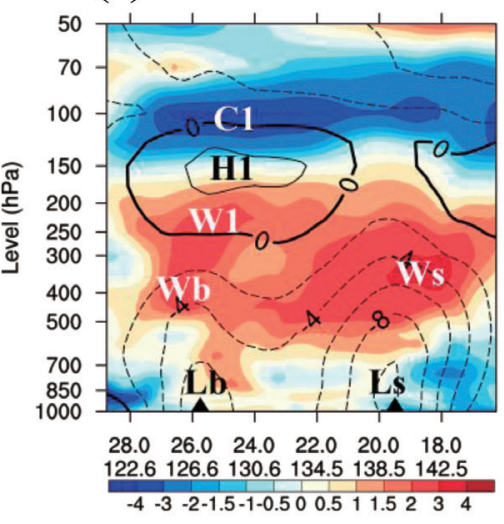

(f)

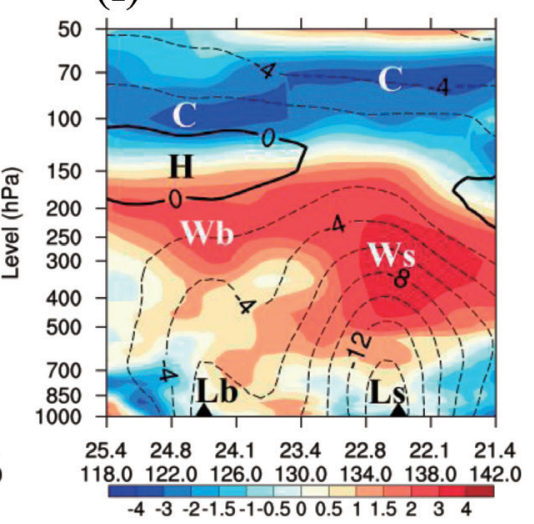

Fig. 2. Vertical sections of height and temperature along the connection line between two TC centers of Bopha and Saomai (2000) at 1200 UTC, 8 September 2000 (upper panel) and 1200 UTC, 9 September 2000 (lower panel). (a, d) Total height (solid line, $200 \times 10 \mathrm{gpm}$ interval) and total temperature (shading, $10 \mathrm{~K}$ interval), (b, e) climatic height (solid line, $200 \times 10 \mathrm{gpm}$ interval) and climatic temperature (shading, $10 \mathrm{~K}$ interval), and (c, f) height anomalies (solid and dashed lines, $2 \times 10$ gpm interval) and temperature anomalies (shading, 0.5 and $1 \mathrm{~K}$ intervals). Letters "Lb" and "Ls" indicate the height anomalous centers of Bopha and Saomai (2000) and letters "Wb" and "Ws" are their temperature anomalous centers, respectively. Letters "H1," "W1," and "C1" are the anomalous system at the upper troposphere. Symbol " $\boldsymbol{\Delta}$ " indicates the position of each TC and longitude-latitude grids are digital below the $\mathrm{x}$-axis.

The vertical structures of the two TCs at 1200 UTC, 8 September 2000 and 1200 UTC, 9 September 2000 are depicted in Fig. 2. The total height and temperature as well as climatic height and temperature are vertically shown, respectively, in Figs. 2a and $2 b$ crossing two central positions of the two TCs (red lines in Fig. 1a). No significant signals can be found indicating the two TCs at 1200 UTC, 8 September 2000. However, the differences between total and climatic fields show two significant negative centers of height anomalies in the surface and two positive centers of temperature anomalies in the upper tropo- sphere right above the two TCs in Fig. 2c. From the troposphere to the stratosphere, hydrostatic balance is satisfied vertically from both height and temperature anomalies. It is clearly noted that a negative center (C1) and a positive center (W1) of temperature anomalies are situated above and below the positive center (H1) of height anomalies at the upper troposphere. Even in the lower troposphere, a positive center $(\mathrm{Wb})$ of temperature anomalies is located above the negative center $(L b)$ of height anomalies at the surface. This hydrostatic balance relationship with obvious and defined positive-negative signals of height and 
(a)
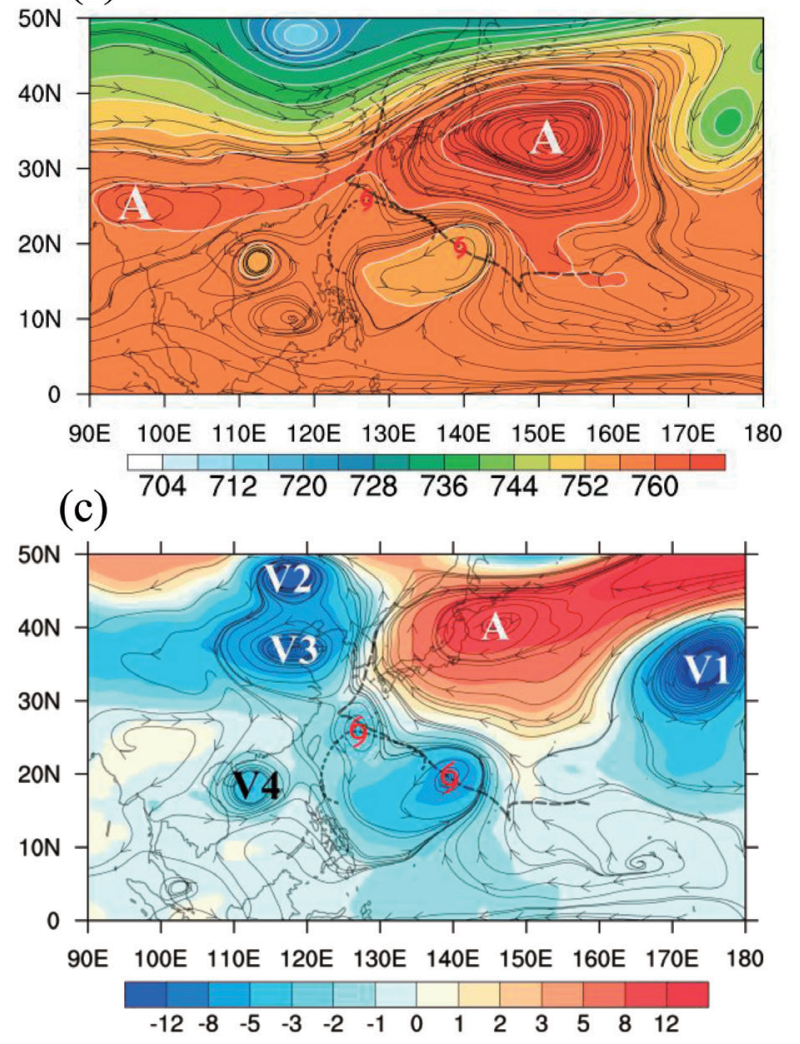

(b)
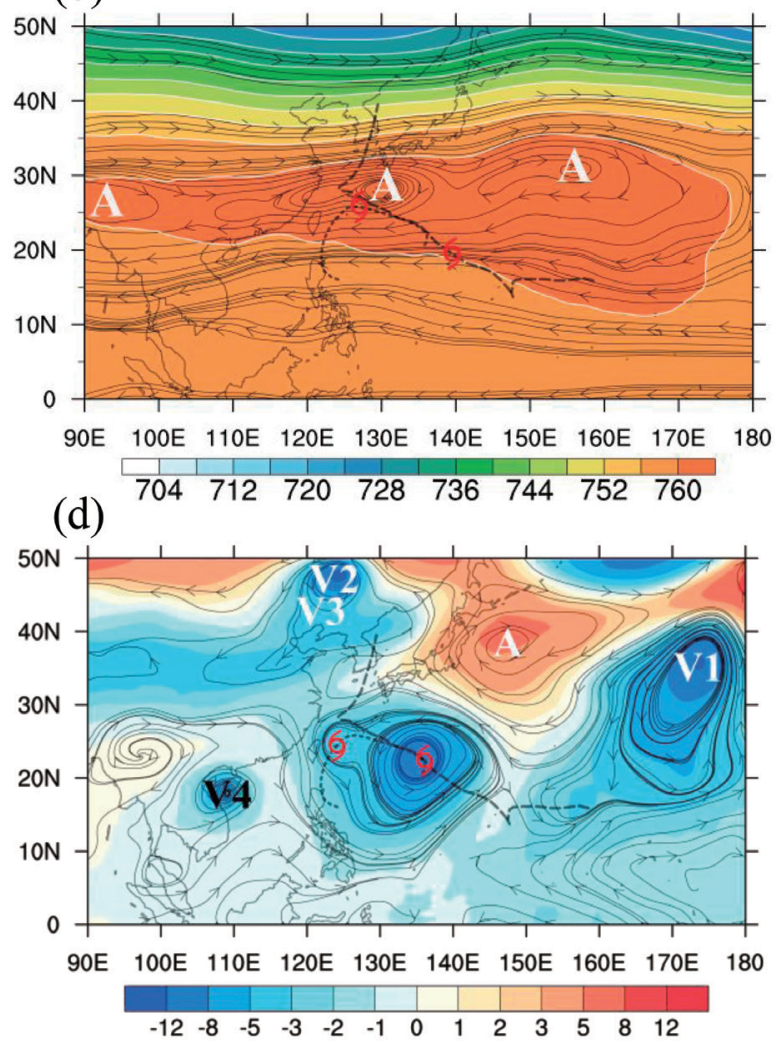

Fig. 3. (a) Total height (shading, $4 \times 10$ gpm interval) and total flow, (b) climatic height (shading, $4 \times 10$ gpm interval) and climatic flow, (c) height anomaly (shading, $1 \times 10,2 \times 10$ and $4 \times 10$ gpm intervals) and anomalous flow at $400 \mathrm{hPa}$ at 1200 UTC, 8 September 2000. (d) Same as (c) but at $450 \mathrm{hPa}$ at $1200 \mathrm{UTC}, 9$ September 2000. Letters "A" and "V" indicate the centers of anticyclone and vortex, respectively. The symbol "6" denotes the position of each TC, and the dashed line is the best track.

temperature anomalies is also observed in Fig. $2 \mathrm{f}$ at 1200 UTC, 9 September 2000, but there is not any signal indicating the two TCs in Figs. $2 \mathrm{~d}$ and $2 \mathrm{e}$ from total height, total temperature, climatic height, and climatic temperature.

$\mathrm{TC}$ is an anomalous circulation system. The movement of TCs is influenced by climatic steering flow and interacts with other anomalous systems in the vicinity (Qian et al. 2014; Huang et al. 2015). Figure $3 \mathrm{a}$ is the total flow that can be decomposed as climatic flow (Fig. 3b) and anomalous flow (Fig. 3c). The strong TC (Saomei) is closer to the subtropical high than that of the weak TC (Bopha), so the track of Saomei was mainly influenced by total flow (Fig. 3a). Under the climatic flow as shown in Fig. 3b, Bopha and Saoma should move westward. However, considering the interaction among two TCs and other anom- alous systems, a complex movement can be observed from Fig. 3c to Fig. 3d. At 1200 UTC, 8 September 2000, an anomalous anticyclone over Japan and four anomalous vortices near the two TCs from East Asia to the NW Pacific existed at the fields of height and wind anomalies at $400 \mathrm{hPa}$ (Fig. 3c).

As shown in Figs. $2 a$ and $2 b$, the deviation of total field from climatic field cannot be directly distinguished, but it is clear from the anomalous field in Fig. 2c. Similarly, the height and flow deviations cannot be identified from the total field in Fig. 3a and the climatic field in Fig. 3b, but their differences or anomalies are clearly shown in Fig. 3c, in which an anomalous anticyclone and four anomalous vortices from East Asia to NW Pacific surrounded two TC vortices. These anomalous systems' locations and intensities changed from day to day. From 1200 UTC, 
8 September 2000 to 1200 UTC, 9 September 2000, the distance of two anomalous TC vortices decreased and Saomai strengthened, but the anomalous anticyclone weakened in the NW Pacific while two mid-latitude vortices (V2 and V3) merged (Figs. 3c, d). Whether there were interactions between the two TCs and other anomalous systems needs to be examined by the GBAM.

The vorticity anomalies, derived from anomalous wind, at vertical section and horizontal plane are shown from Figs. 4a to 4d at 1200 UTC, 8 September 2000 and 1200 UTC, 9 September 2000, respectively. The significant positive vorticity anomalies corresponded well to the two TCs extending from the surface to the upper troposphere (Figs. 4a, b). There was a maximum center of positive vorticity anomalies at around $400-500 \mathrm{hPa}$, and thus we plot the horizontal distribution of vorticity anomalies at 400 and $450 \mathrm{hPa}$, respectively, at $1200 \mathrm{UTC}, 8$ September 2000 and 1200 UTC, 9 September 2000 (Figs. 4c, d). Near the two TC vortices there was another anomalous vortex (a large center of positive vorticity anomalies) in the SCS. The two TC centers circled each other in the counterclockwise direction, as can be observed from the red line in Figs. $4 \mathrm{c}$ and $4 \mathrm{~d}$.

To explain whether there was a binary interaction between two nearby TCs, comparison experiments are shown in Figs. $4 \mathrm{e}$ and $4 \mathrm{f}$ as examples. The positive vorticity anomaly around the Bopha vortex (weak one) in the East China seas is replaced by climatic vorticity at 1200 UTC, 8 September 2000 (Fig. 4e). Similarly, the positive vorticity anomaly around the Saomei vortex (strong one) is replaced by climatic vorticity at 1200 UTC, 9 September 2000 (Fig. 4f). To examine whether the two TCs interacted, the GBAM is used to compare their tracks of prediction over the following $48 \mathrm{~h}$ when initiated at 1200 UTC 7, 1200 UTC 8, 0000 UTC 9, and 1200 UTC 9 September 2000 , respectively. Track deviation is qualitatively examined but their speed deviation is not considered in Fig. 5. The black line ("Prediction") is the track predicted by the GBAM, while the green line ("Comparison") is the predicted track when another TC vortex of positive vorticity anomalies is replaced by climatic vorticity. If the black and green lines are consistent to a TC best track (red line), it means that another TC does not influence on this TC's track. If the two predicted lines show a large deviation in distance, it means that another TC directly influences this TC's track. If large deviations of the two lines are found in both TCs' predictions, it means that there is a binary interaction between two TCs.
In this case, the track of Saomai is not influenced by the Bopha vortex, but the track of Bopha is influenced by Saomai, particularly from 24 to $48 \mathrm{~h}$, when their prediction is initiated from 1200 UTC, 7 September 2000 (Fig. 5a). It is noted from point "A" that the prediction (black line) and comparison (green line) tracks of Saomai follow the best track (red line) from 24 and $48 \mathrm{~h}$. From point "B" the prediction track is curved to the best track of Bopha, but the comparison shows a rapid return. Starting from 1200 UTC, 8 September 2000 (Fig. 5b), the two TCs had no significant interaction. At the other two initial times of 0000 and 1200 UTC, 9 September 2000, the predicted track of Saomai are accurate in the first $24 \mathrm{~h}$ in both cases with and without Bopha, but the predicted track of Bopha is different if Saomai's vortex is removed (Figs. 5c, d). This implies that Bopha was influenced by Saomai starting from 1200 UTC, 7 September 2000 when Bopha approached Taiwan Island, but the latter was not influenced by the former. Using the potential vorticity diagnosis, Wu et al. (2003) described that Bopha moved southward due to the binary interaction with Saomai. However, the dynamical model showed that the southward movement of Bopha was only influenced by Saomai, but without any binary interaction.

\subsection{Goni and Morakot (2009)}

The TC Morakot (2009) caused severe damage when it hit Taiwan Island on 7 August 2009, so we give a detailed analysis in this subsection. Wu et al. (2012) reported that the two TCs (Goni and Morakot) had an interaction during their common lifetime period. Figure 6 shows the vertical sections of height and temperature anomalies along the connection of the two TC centers at 0000 and 1200 UTC, 6 August 2009. The anomalous vortex of Morakot has a depth of $250 \mathrm{hPa}$, while that of Goni is a relatively shallow $300 \mathrm{hPa}$. The hydrostatic balance relationship is also clearly observed in Figs. $6 \mathrm{a}$ and $6 \mathrm{~b}$ for the anomalous Morakot vortex. A negative center $(\mathrm{Cl})$ and a positive center $(W l)$ of temperature anomalies are situated above and below, respectively, the positive center (H1) of height anomalies at the upper troposphere. In the lower troposphere, a positive center $(\mathrm{Wm})$ of temperature anomalies is located above the negative center $(\mathrm{Lm})$ of height anomalies at the surface. It means that the temperature anomalies can be derived from the height anomalies based on the hydrostatic balance relationship.

The intensity evolution of the two TCs is compared from their vertical-time sections of height anomalies 
(a)

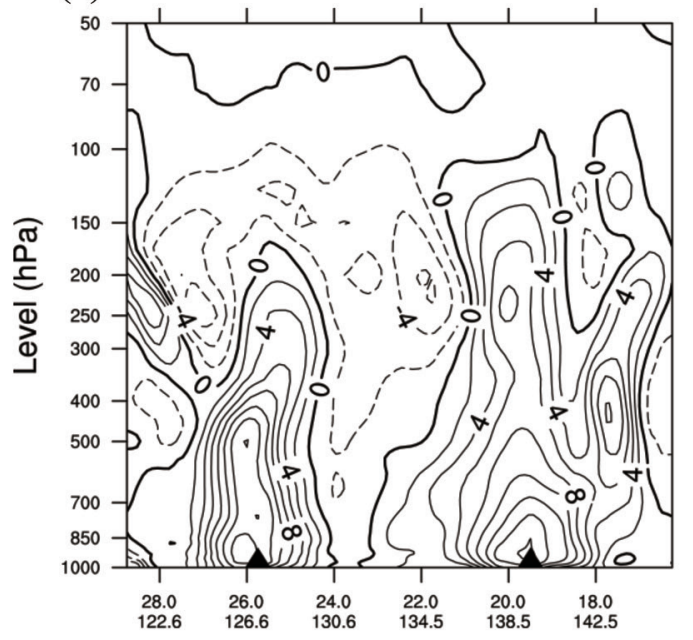

(c)

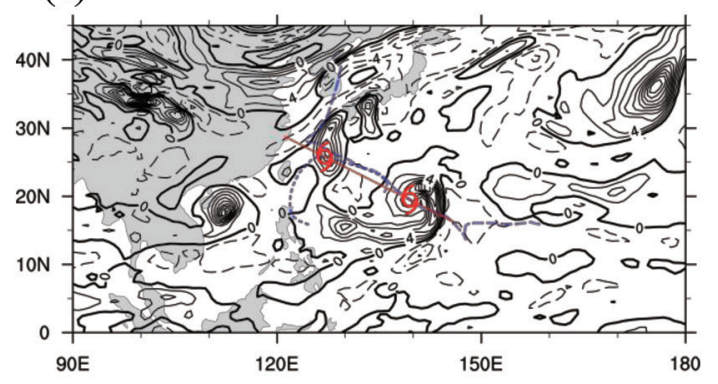

(e)

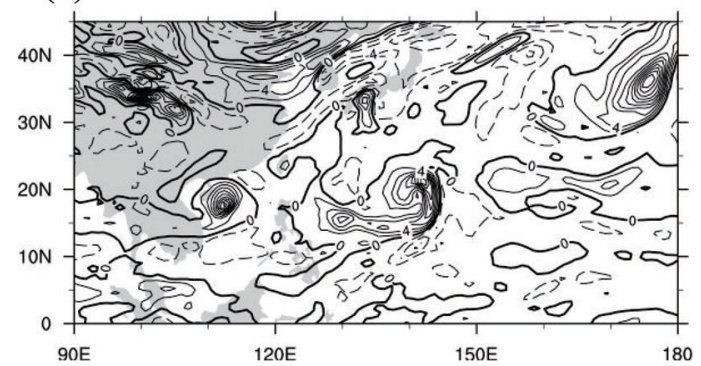

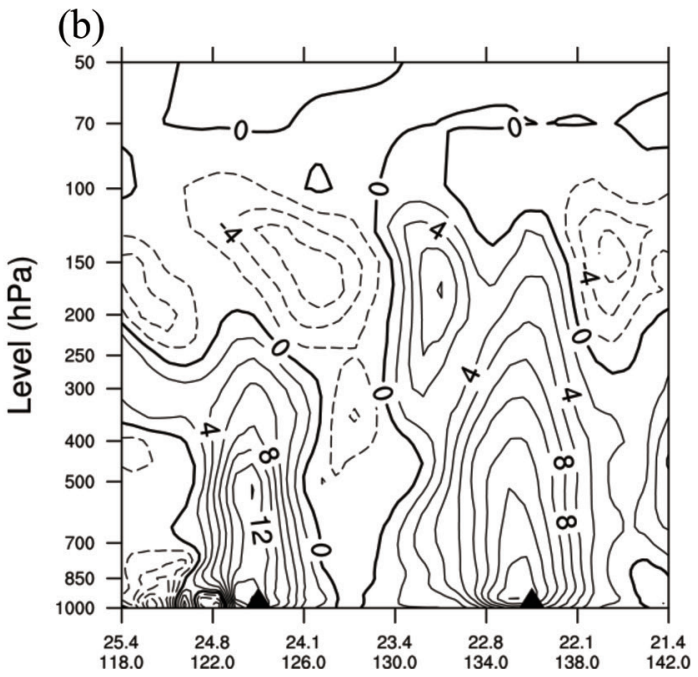

(d)

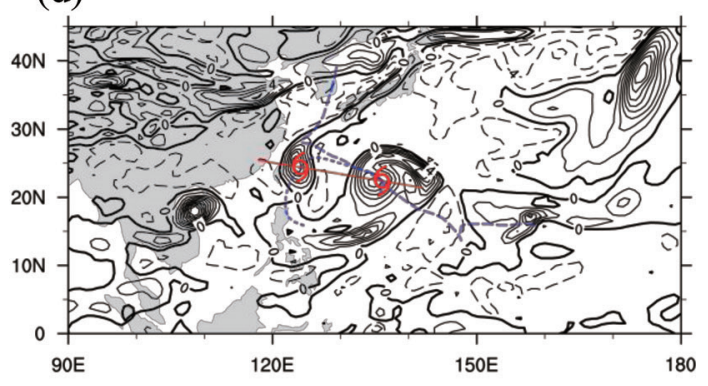

(f)

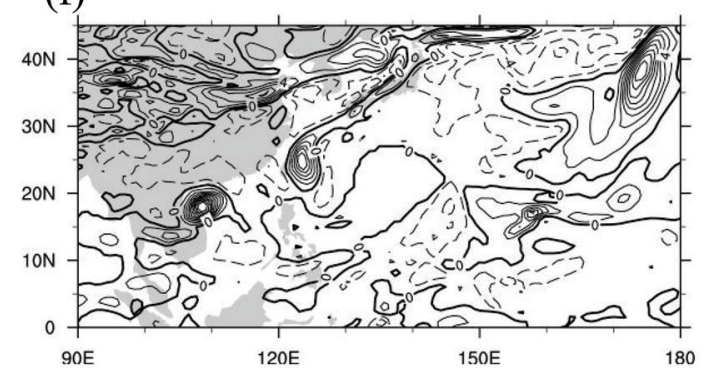

Fig. 4. Vorticity anomalies $\left(10^{-5} \mathrm{~s}^{-1}\right)$ in the vertical section along the connection of two TC centers at (a) 1200 UTC, 8 September 2000 and (b) 1200 UTC, 9 September 2000 . Vorticity anomalies $\left(10^{-5} \mathrm{~s}^{-1}\right)$ at (c) $400 \mathrm{hPa}$ at 1200 UTC, 8 September 2000 and at (d) $450 \mathrm{hPa}$ at 1200 UTC, 9 September 2000. Vorticity anomalies in (e) and (f) are the same as in (c) and (d) except the positive vorticity anomalies around the Bopha and the Saomei vortices are replaced by climatic vorticity.

and temperature anomalies in Fig. 7. The strongest center of Goni with the value $-120 \mathrm{gpm}$ occurred at 0000 UTC, 7 August 2009 (Fig. 7a), and the strongest center of Morakot with the value $-240 \mathrm{gpm}$ happened at 0000 UTC, 8 August 2009 (Fig. 7b). Negative temperature anomalies sometimes appeared during their lifetime periods in the lower layer. Positive temperature anomalies always existed in the upper troposphere centered around $250-300 \mathrm{hPa}$.

Besides the two positive centers of vorticity anomalies near the surface, another two positive centers of vorticity anomalies can be found at around 400 
(a)

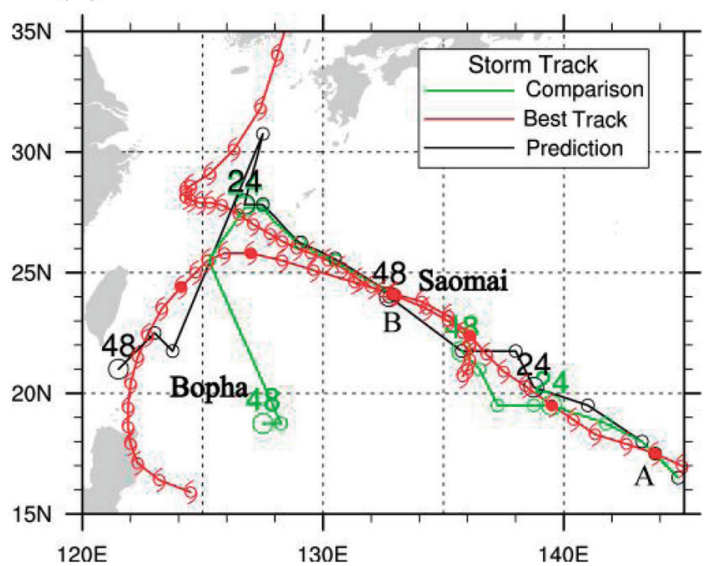

(c)

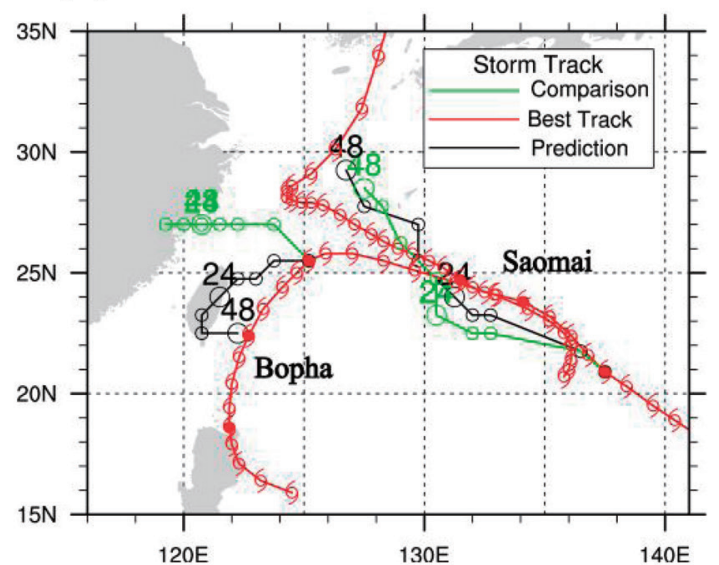

(b)

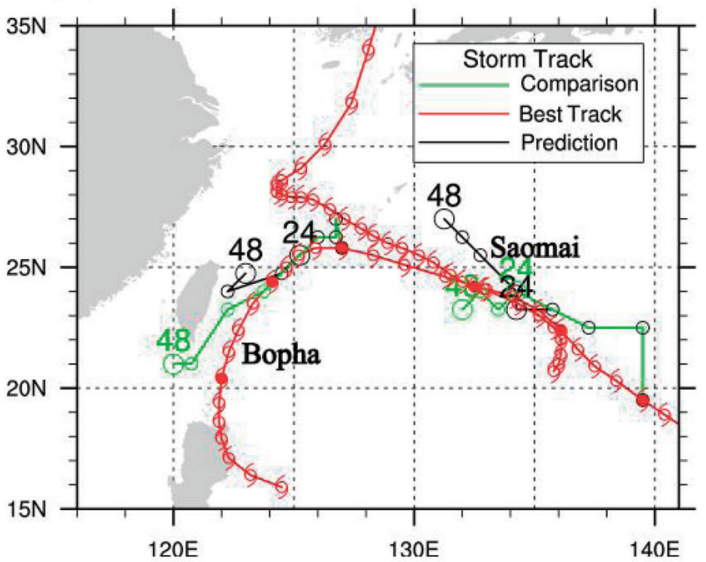

(d)

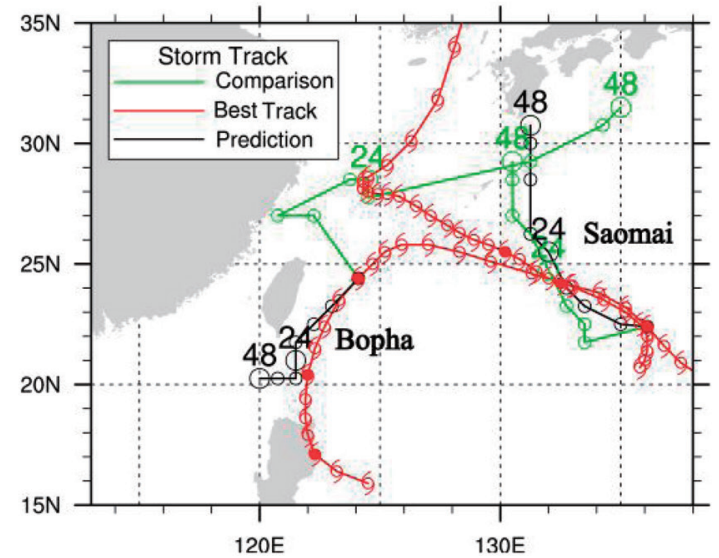

Fig. 5. Best track (red line) and original GBAM prediction track (black line), and compared prediction (comparison) track (green line) when another TC vortex is replaced by climatic flow initiated at (a) $450 \mathrm{hPa}$ at $1200 \mathrm{UTC}, 7$ September 2000, (b) $400 \mathrm{hPa}$ at 1200 UTC, 8 September 2000, (c) $600 \mathrm{hPa}$ at $0000 \mathrm{UTC}, 9 \mathrm{September} 2000$, and (d) $450 \mathrm{hPa}$ at 1200 UTC, 9 September 2000. The highlighted symbol "9" and the numbers 24 and 48 indicate each TC following the best track and model prediction for the following 24 and $48 \mathrm{~h}$. In (a), the two letters "A" and "B" are the two location points of Saomei and Bopha at the model initial time.

hPa over the two TCs (Fig. 8a). It is clear that there were several positive and negative centers of vorticity anomalies in the vicinity of the two TCs at $400 \mathrm{hPa}$ (Fig. 8b). Thus, the anomaly-based analysis and the GBAM are conducted to figure out whether these anomalous systems directly interact with each other. During the common lifetime period of the two TCs, anomalous flows and height anomalies changed with time and altitude. At $450 \mathrm{hPa}$ at $0000 \mathrm{UTC}, 6$ August 2009, Morakot was located in the eastern sea of Taiwan Island and directly influenced by the total steering flow of the NW Pacific subtropical high, while Goni was making landfall on the South China coast without obvious total steering flow (Fig. 9a). The two TCs were situated in a climatic easterly flow (Fig. 9b). An anomalous anticyclone was located in the NW Pacific and another weak anomalous anticyclone was in the central SCS, while at the same time there was an anomalous vortex (V1) in the southeast of Morakot (Fig. 9c). At $450 \mathrm{hPa}$ at $1200 \mathrm{UTC}$, 6 August 2009, Morakot propagated closer to Taiwan Island in the east sea and Goni remained at the same position. The NW Pacific anomalous anticyclone strengthened and extended westward, but the anoma- 
(a)

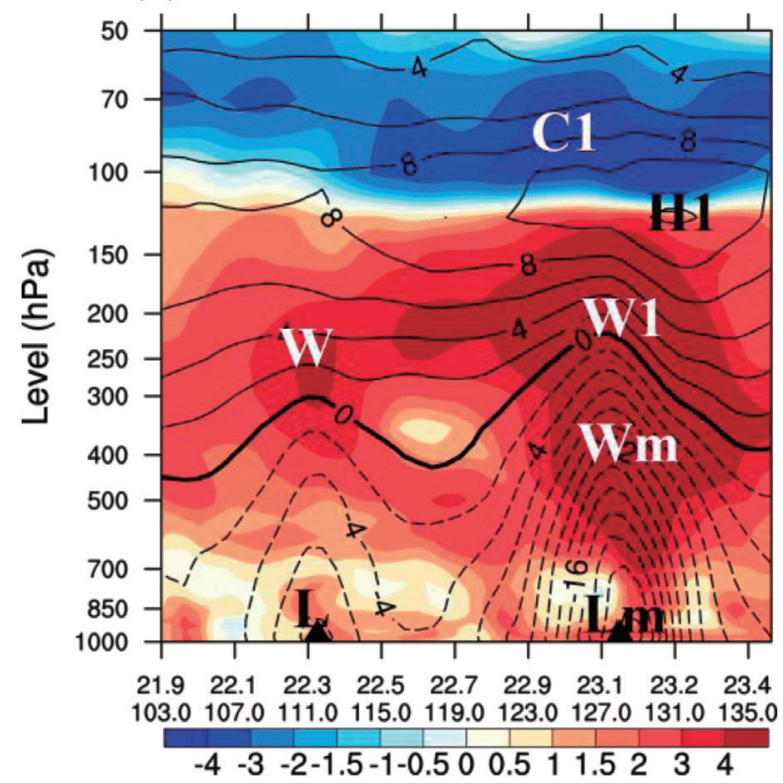

(b)

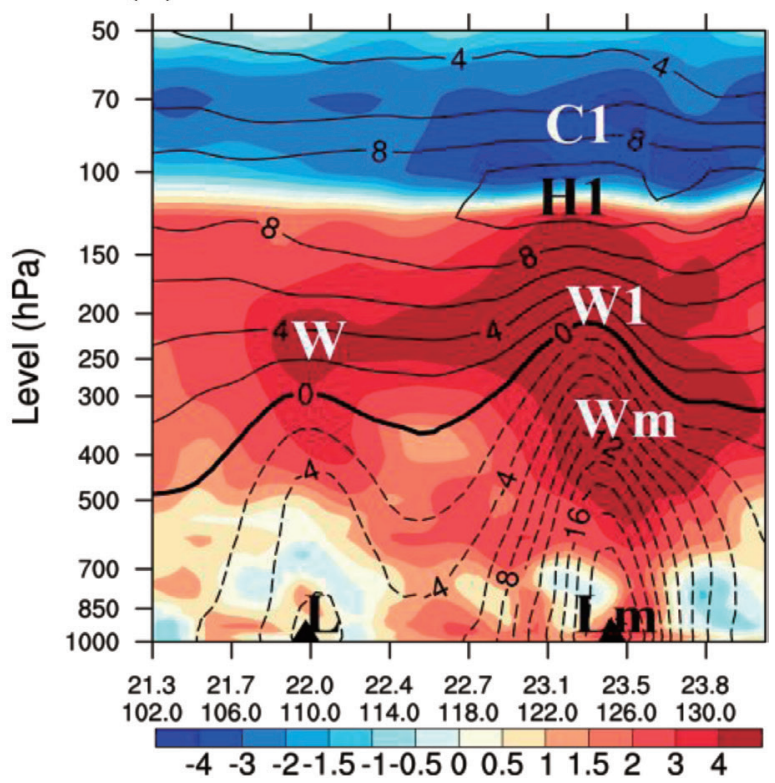

Fig. 6. Same as in Figs. 2c and 2f, except vertical sections of height anomalies (solid and dashed lines, $2 \times 10$ gpm interval) and temperature anomalies (shading, 0.5 and $1 \mathrm{~K}$ intervals) along the connection of the two TCs (Goni and Morakot) at (a) 0000 and (b) 1200 UTC, 6 August 2009.

lous anticyclone in the central SCS had moved to the Indo-China Peninsula, while the two TCs and another anomalous vortex (V1) oriented from the South China coast to the NW Pacific (Fig. 9d).

The GBAM shows that there is no binary interaction between the two TCs before Morakot made landfall on Taiwan Island. As illustrated in Figs. 10a to $10 \mathrm{~d}$, the black and green lines show consistency for the model prediction that initiated from 1800 UTC, 4 August 2009, 0000 UTC, 6 August 2009, 0600 UTC, 6 August 2009, and 1200 UTC, 6 August 2009. However, deviations become large after replacing the anomalous anticyclone $(A 1)$ with the climatic flow at 1800 UTC, 4 August 2009, 0000 UTC, 6 August 2009, 0600 UTC, 6 August 2009, and 1200 UTC, 6 August 2009 (Figs. 10e, h). The green line turns southward in Fig. 10f, but it returns eastward in Figs. $10 \mathrm{~g}$ and $10 \mathrm{~h}$. The model experiment shows that the right-turning track of Morakot is caused by the anomalous anticyclone system in the NW Pacific when it is approaching Taiwan Island. This result is different from previous studies. In traditional methods, the combination of the climatic NW Pacific circulation in Fig. 9b and the anomalous anticyclone "A1" in Fig. $9 \mathrm{c}$ is used as the total steering flow to influence the TC movement. Using an MM5 model, Wu et al. (2012) carried out different modeling experiments with different TC intensities and sizes. Their result showed that Morakot had a greater impact on the motion of Goni, while Goni had a relatively limited impact on Morakot. The uncertainty of previous work presents a difficulty in obtaining the intensity and size of TCs.

\subsection{Two further pairs of TCs}

Similarly, another two TC pairs, Fengshen and Fungwong (2002), as well as Bopha and Saomai (2006), are analyzed and modeled in this subsection. The hydrostatic balance relationship is also vertically observed in Figs. 11a and 11b for the two TCs and other anomalous systems. The positive centers of temperature anomalies at the upper troposphere satisfy the hydrostatic balance with TC vortices in the lower layer and anomalous anticyclone systems in the upper layer. The large center of vorticity anomalies is vertically observed around $500 \mathrm{hPa}$ so the horizontal distribution of vorticity anomalies at $500 \mathrm{hPa}$ is plotted in Figs. 11c and 11d. At 0000 UTC, 22 July 2002, the Fungwong vortex was stronger than the Fengshen vortex indicated by the vorticity anomalies 
(a)

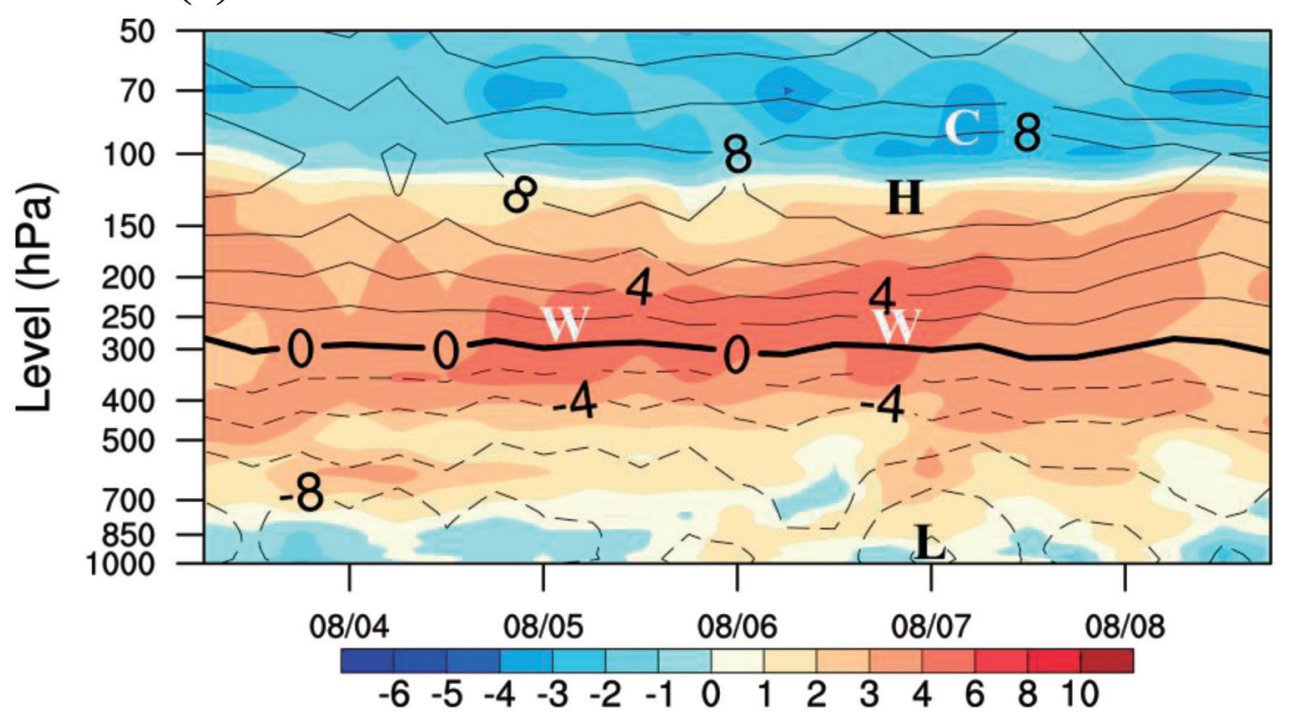

(b)

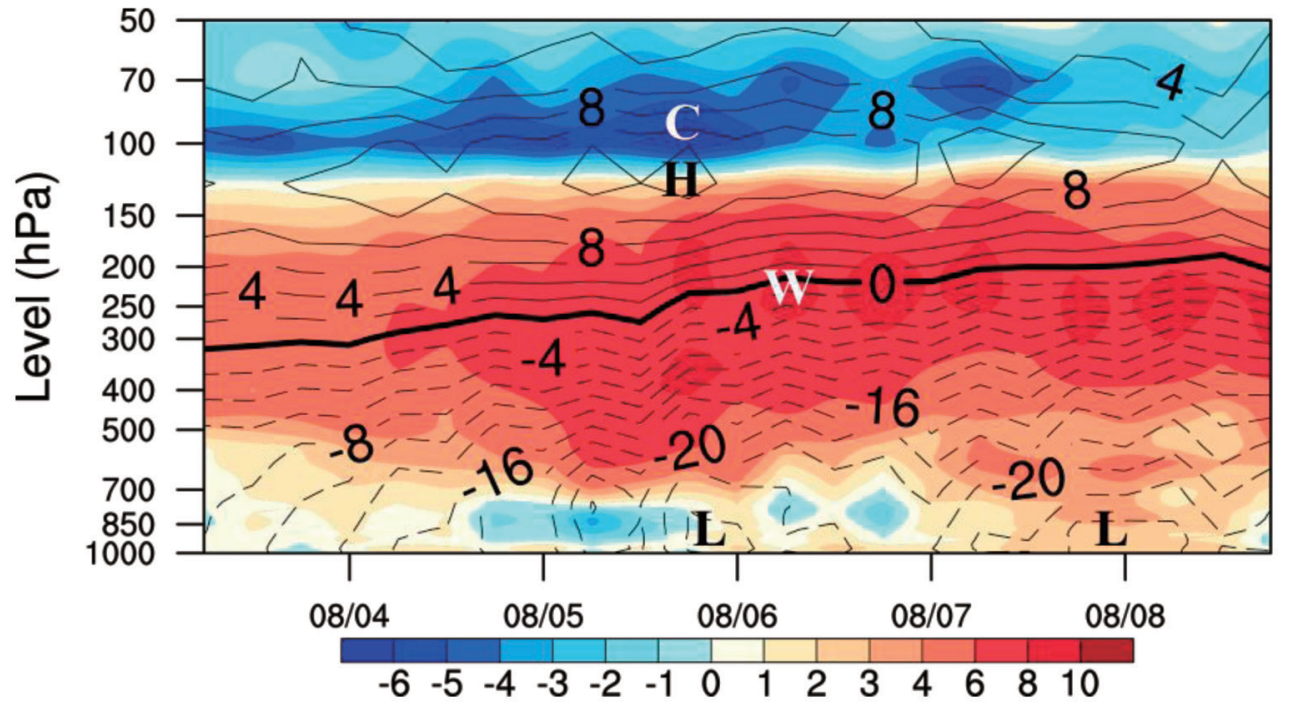

Fig. 7. Vertical-time sections of height anomalies (solid and dashed lines, $2 \times 10$ gpm interval) and temperature anomalies (shading, 1 and $2 \mathrm{~K}$ intervals) for (a) Goni and (b) Morakot in their common lifetime period from 0600 UTC, 3 August 2009 to 1800 UTC, 8 August 2009.

with a relatively larger size (Fig. 11c). At 0000 UTC, 24 July 2002, their distance decreased (Fig. 11d). The GBAM showed that Fengshen had an influence on the movement of Fungwong, but the latter did not directly influence the former's movement (Fig. 12). The GBAM can accurately predict the straight track of Fengshen whether Fungwong exists or not. The circular movement of Fungwong is also accurately predicted by the GBAM, but it is influenced by Fengshen's circulation. It is noted that if the Fengshen vortex is replaced with a climatic steering flow, the predicted track (green line) of Fungwong is expected northward instead of circular movement. The potential vorticity diagnosis indicated that there was the 

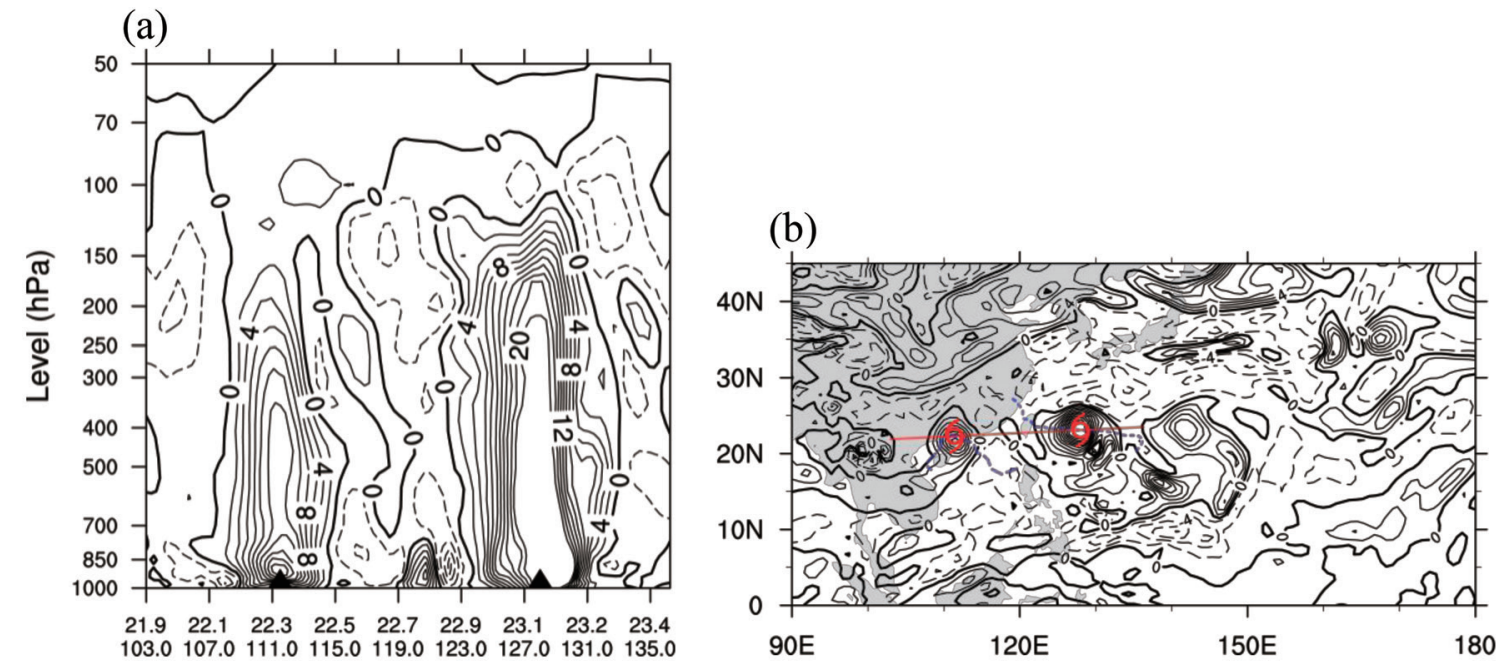

Fig. 8. Same as in Fig. 4, except the two TCs of Goni and Morakot at 0000 UTC, 6 August 2009. In (b) the level is at $400 \mathrm{hPa}$.

(a)
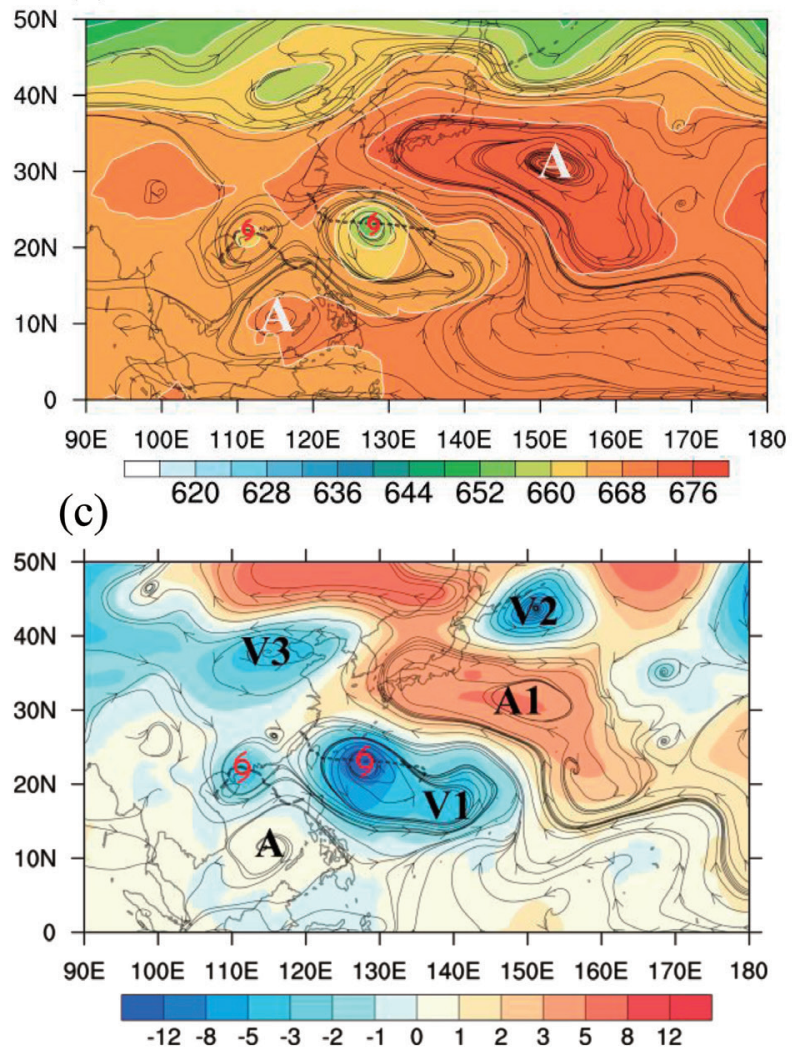

(b)

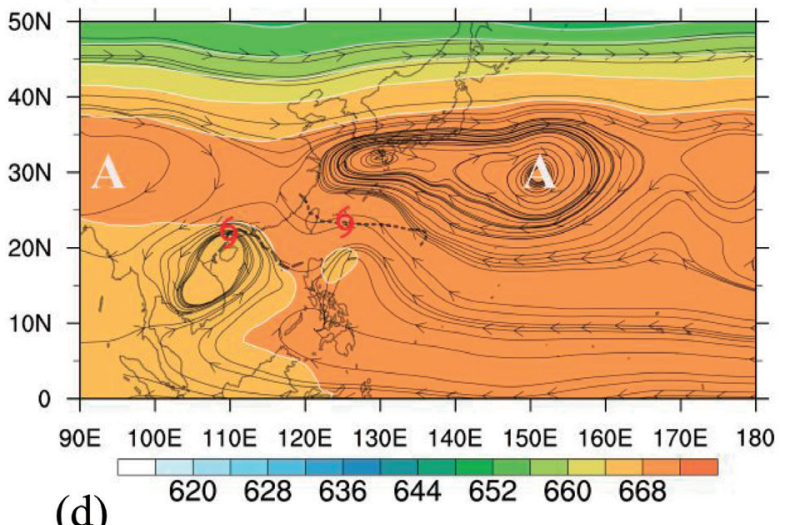

(d)

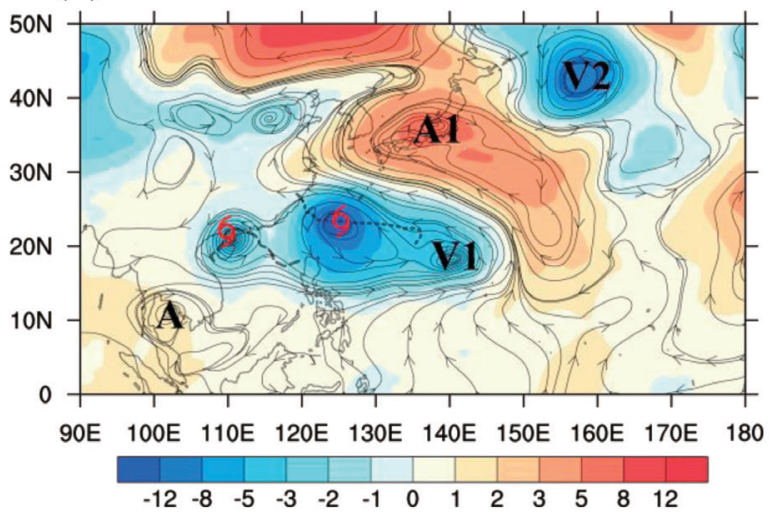

Fig. 9. Same as Fig. 3, except (a, c) at 0000 UTC, 6 August 2009 at $450 \mathrm{hPa}$ and (b, d) at $1200 \mathrm{UTC}, 6$ August 2009 at $450 \mathrm{hPa}$. 
(a)

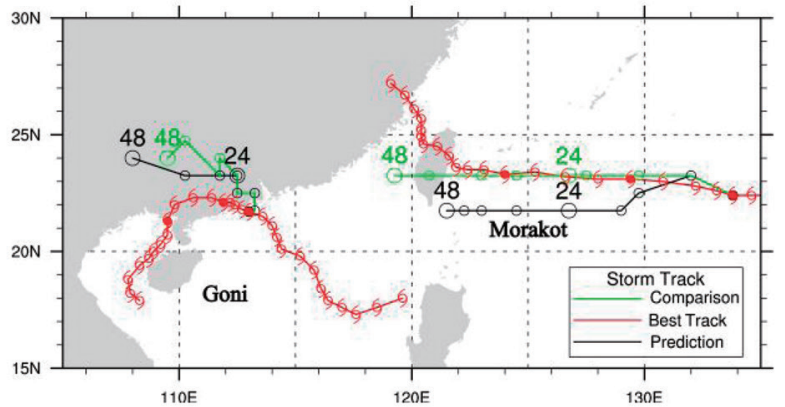

(c)

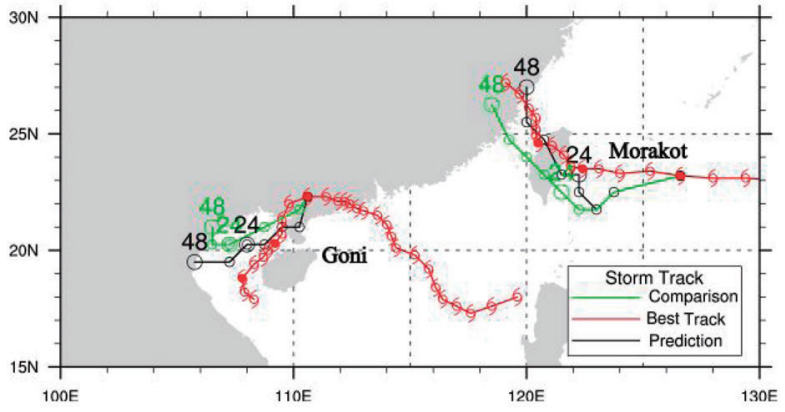

(e)

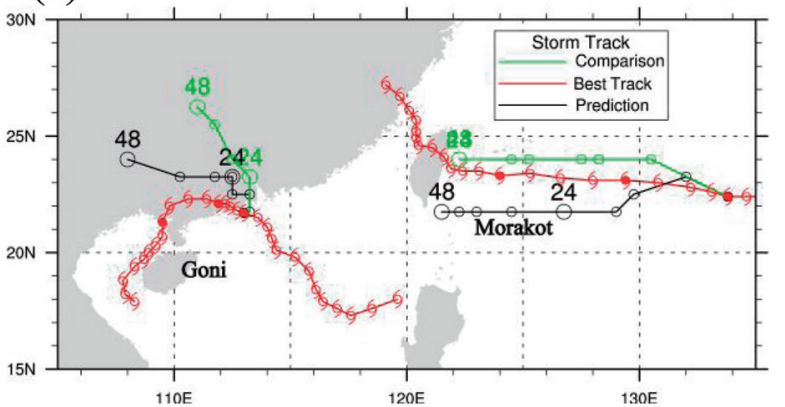

(g)

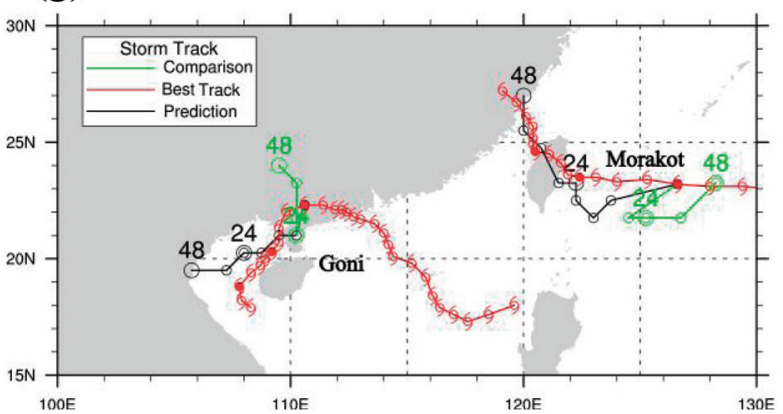

(b)

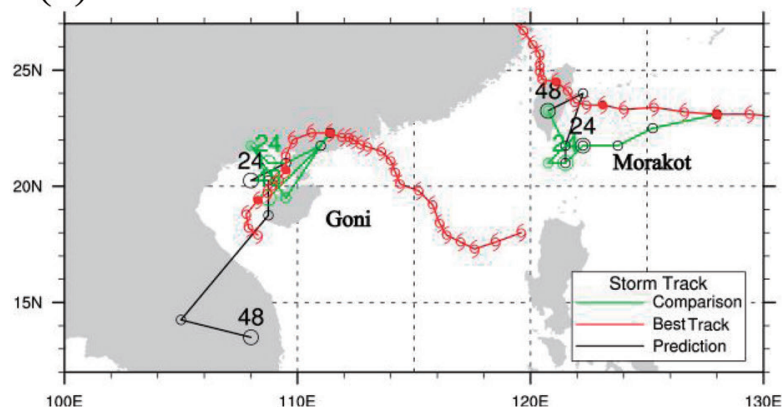

(d)

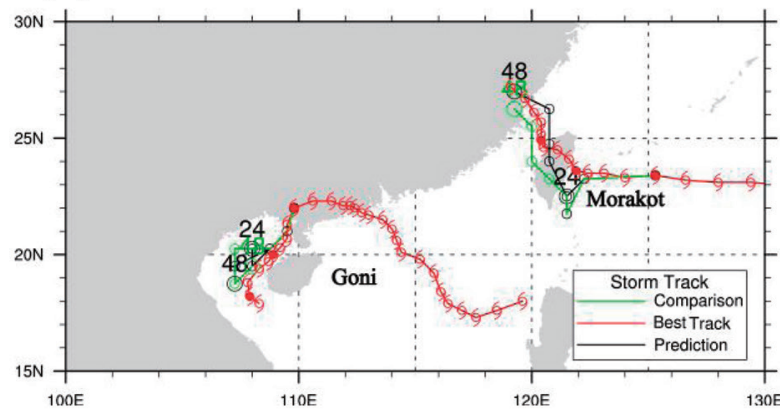

(f)

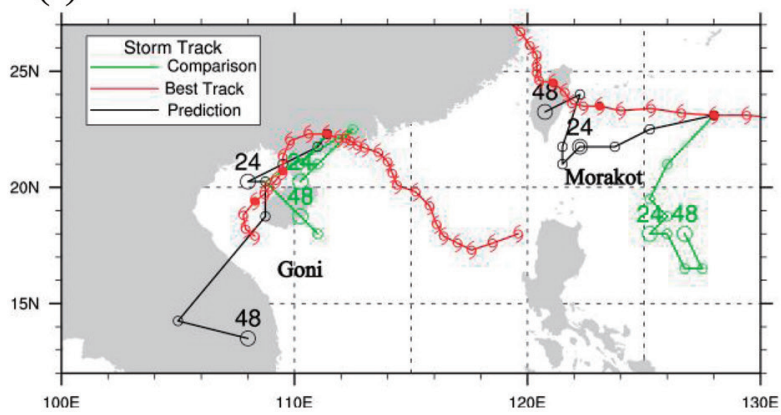

(h)

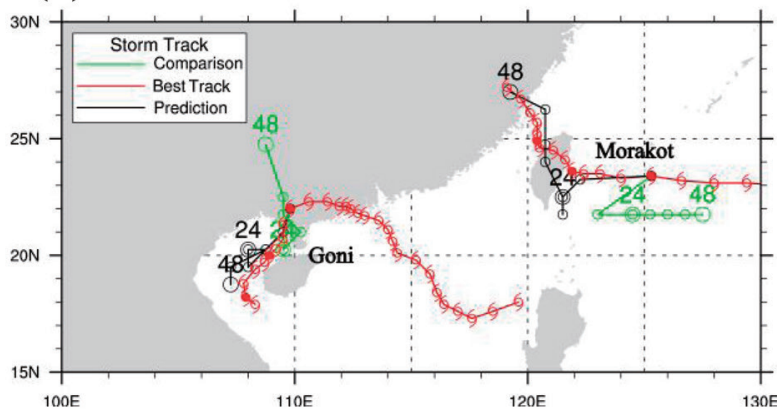

Fig. 10. Same as in Fig. 5, except for the two TCs (Goni and Morakot) initiated respectively at (a) 1800 UTC, 4 August 2009, (b) 0000 UTC, 6 August 2009, (c) 0600 UTC, 6 August 2009, and (d) 1200 UTC, 6 August 2009 at $400,400,450$, and $450 \mathrm{hPa}$, respectively, as well as the movement of two TCs (Goni and Morakot) after replacing the anomalous anticyclone as the climatic flow initiated respectively at (a) 1800 UTC, 4 August 2009, (b) 0000 UTC, 6 August 2009, (c) 0600 UTC, 6 August 2009, and (d) 1200 UTC, 6 August 2009. 
(a)

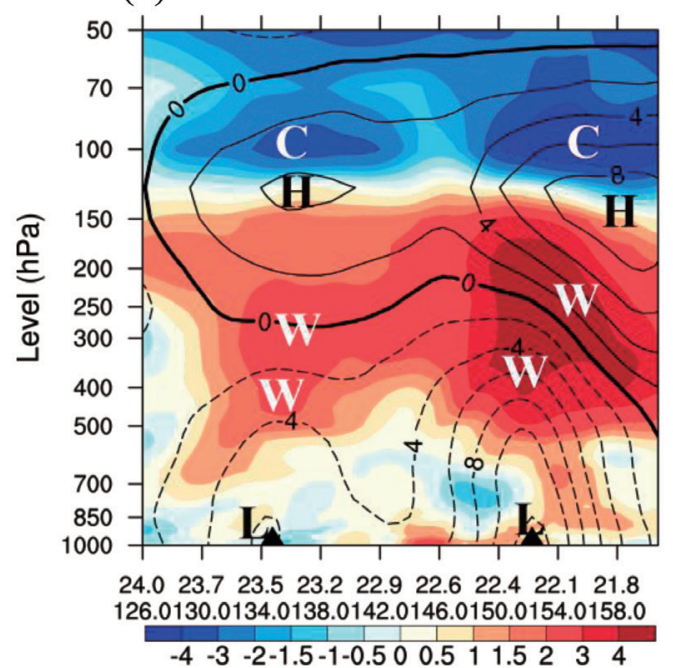

(c)

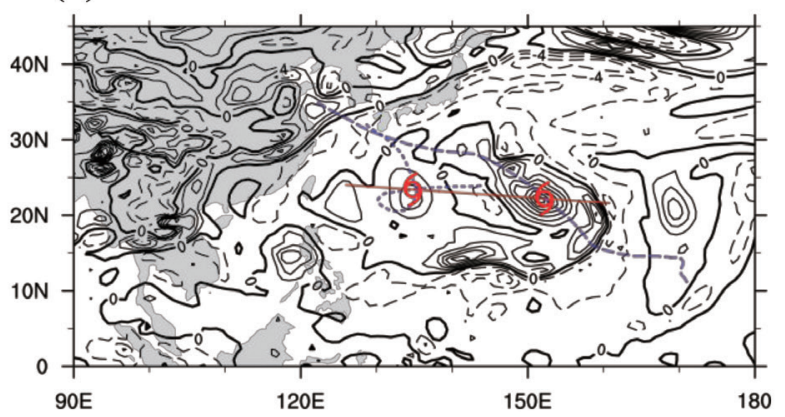

(b)

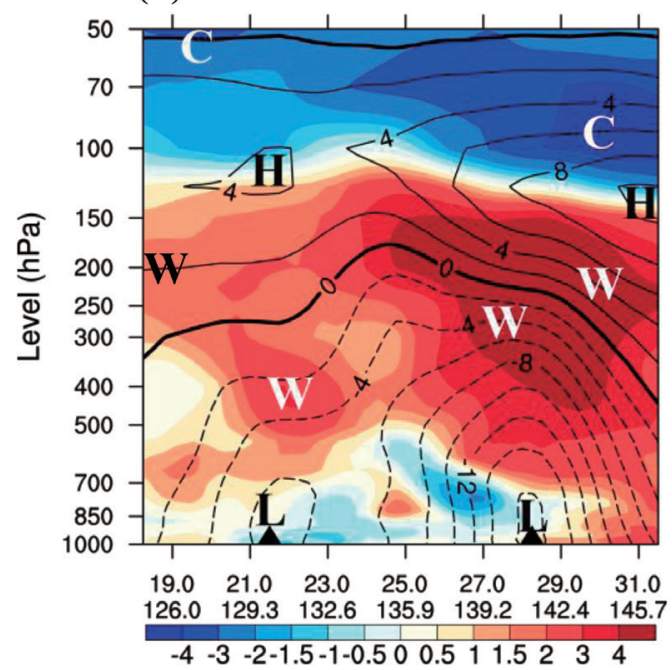

(d)

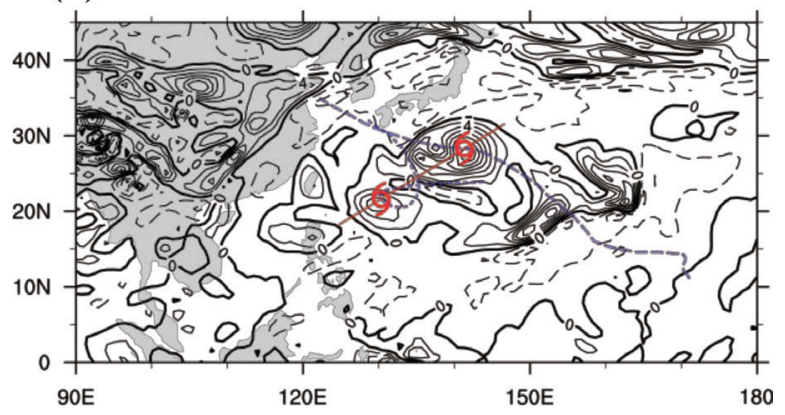

Fig. 11. Same as in Fig. 6, except (a) 0000 UTC, 22 July 2002 and (b) 0000 UTC, 24 July 2002 for the two TCs of Fengshen and Fungwong. Panels (c) and (d) are the same as in Fig. 8b, except at 0000 UTC, 22 July 2002 and 0000 UTC, 24 July 2002 along the connection between two centers of Fengshen and Fungwong at $500 \mathrm{hPa}$.

binary interaction between Fengshen and Fungwong (Yang et al. 2008). However, our result from the GBAM showed that it cannot be referred to as a binary interaction because only the Fengshen affected the movement of Fungwong; the reverse is not true.

The hydrostatic balance relationship is also vertically satisfied in Figs. 13a and 13b for the two TCs of Bopha and Saomai (2006). Saomai was gradually strengthened, particularly since 8 August 2006. This intensification can also be compared in Figs. 13c and $13 \mathrm{~d}$ from their vorticity anomalies at 450 and $500 \mathrm{hPa}$ respectively at 1200 UTC, 7 August 2006 and 0000 UTC, 9 August 2006. The GBAM showed that there was no interaction at 0000 and 1200 UTC, 7 August 2006 when the intensity of Saomai was relatively weaker (Figs. 14a, b). The GBAM could accurately predict the movement in terms of direction for the six initial times. The last four panels showed that Saomai had a direct influence on the movement of Bopha, but the reverse was not true. From Fig. 14c to Fig. 14f, the model predicted that Bopha moved northward (green line) instead of the best track (red line) and the original GBAM prediction (black line) for westward movement. This was the result using the climatic flow to replace the Saomai vortex.

\section{Conclusions and discussion}

Many previous studies indicated that the binary interaction of two nearby TCs can cause unusual tracks and is thus difficult to predict in practice. Four pairs of TCs in the vicinity of the NW Pacific have been studied by previous investigators who 
(a)

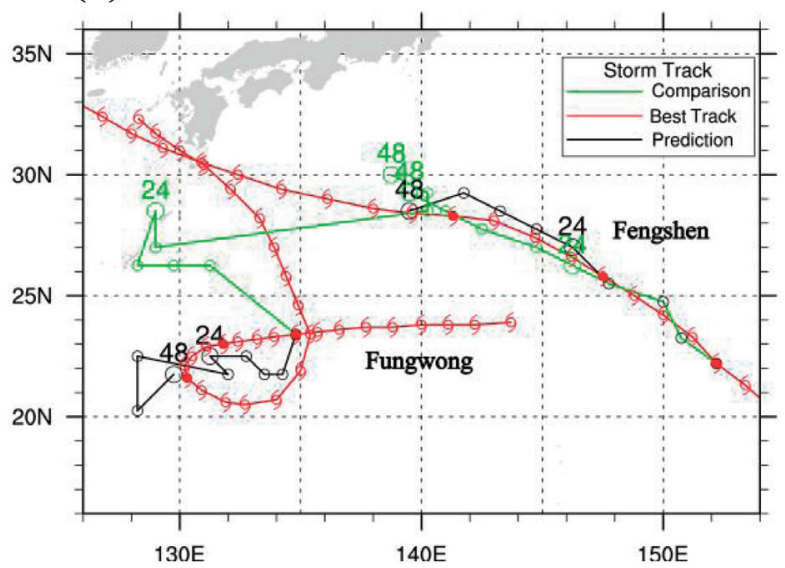

(c)

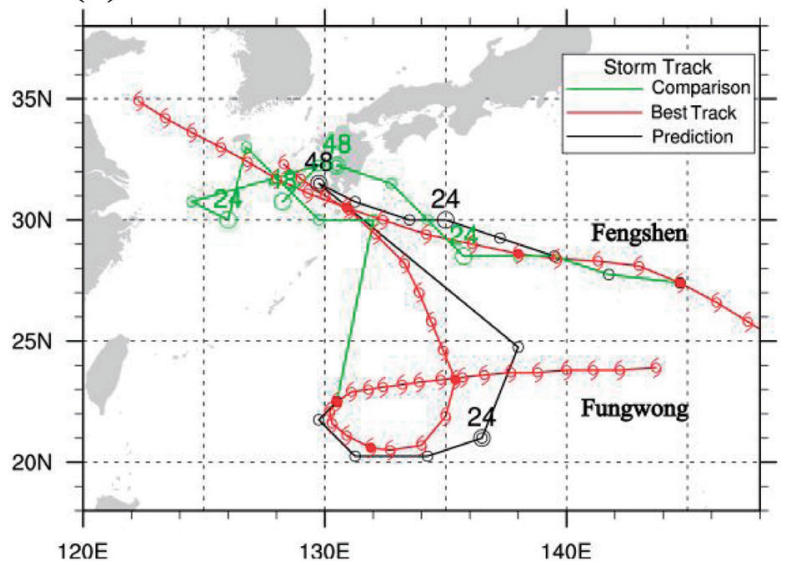

(b)

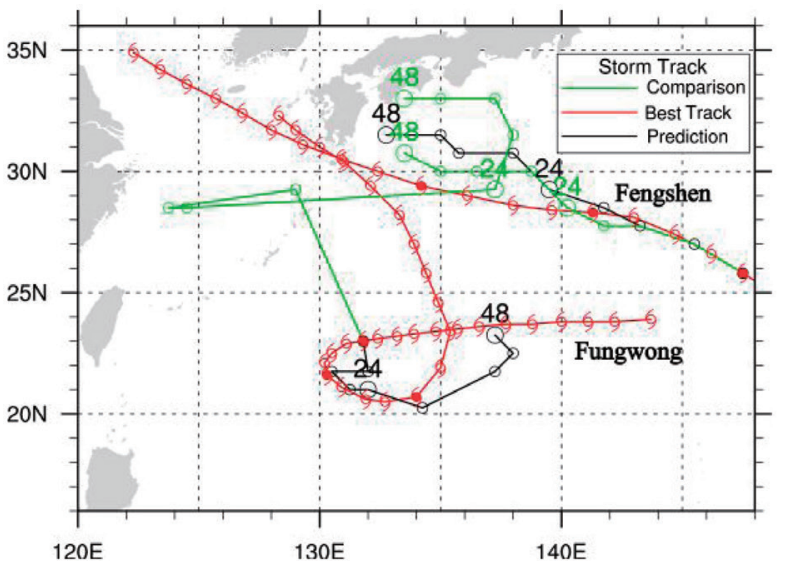

(d)

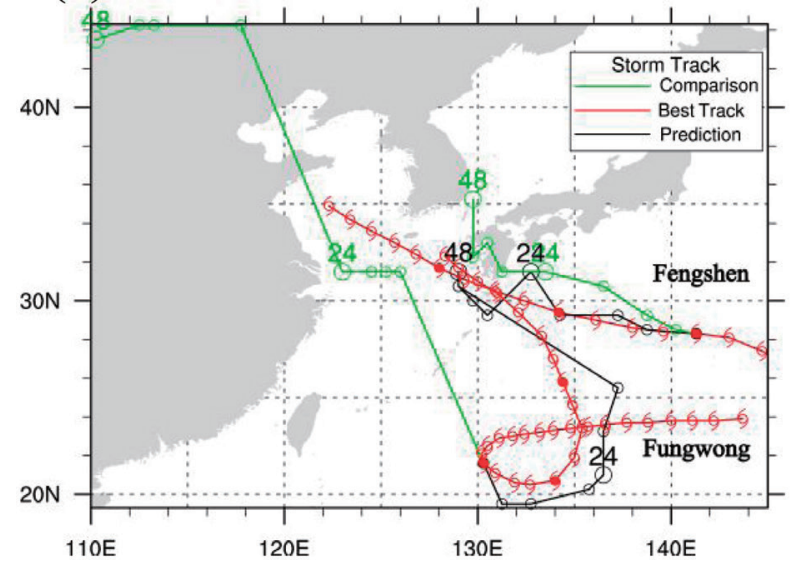

Fig. 12. Same as in Fig. 5 but for the two TCs of Fengshen and Fungwong at (a) 0000 UTC 22, (b) 0000 UTC 23, (c) 1200 UTC 23, and (d) 0000 UTC 24 July 2002 at $500 \mathrm{hPa}$.

found them to have binary interactions. Thus, they are analyzed using the decomposition method and predicted by the GBAM in this paper. All dynamical models for studying whether there is a binary interaction of two vicinity TCs depend on how to extract the TC vortex and surrounding flow from the total flow. However, it is difficult to reasonably define a TC vortex from its intensity and size. This paper decomposes a total wind into a climatic component and an anomaly. The anomaly can be directly used to measure the intensity and size of a TC and other disturbances in the vicinity. Then, the track of TC as an anomalous vortex is influenced by the climatic steering flow and interacts with other anomalous systems in the vicinity. Thus, binary interactions between two TCs as well as interactions between a TC and other anomalous systems can be examined and identified by the GBAM at the optimal level near the maximum center of vorticity anomalies.

Our results show that all four pairs of TCs did not experience binary interactions. In 2000, Bopha's southward motion was considered as the binary interaction with Saomai by using the potential vorticity diagnosis. However, the dynamical GBAM model showed that the southward movement of Bopha (2000) was influenced by Saomai (2000), but no binary interaction was detected. Using the potential vorticity diagnosis, the binary interaction was also considered to happen between Fengshen (2002) and Fungwong (2002). However, the dynamical GBAM model showed that it cannot be referred to as a binary interaction because although Fengshen had an influ- 
(a)

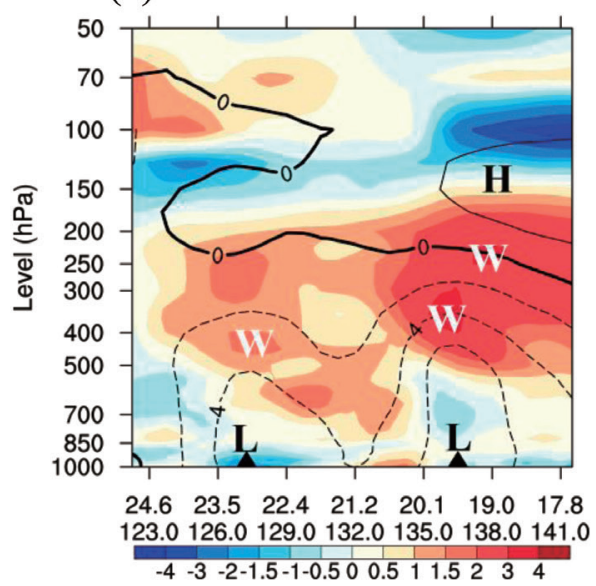

(c)

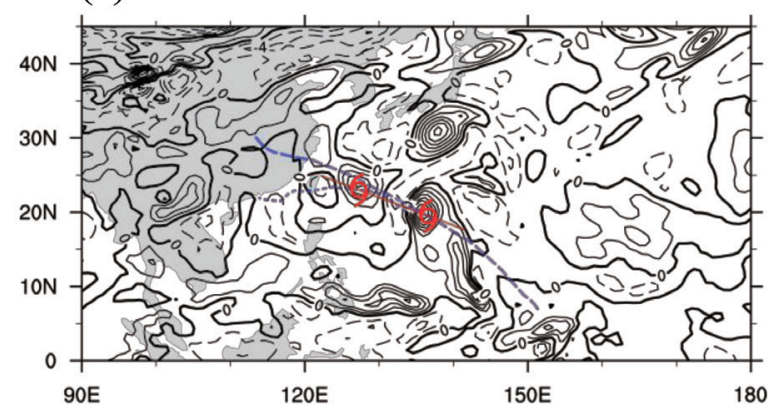

(b)

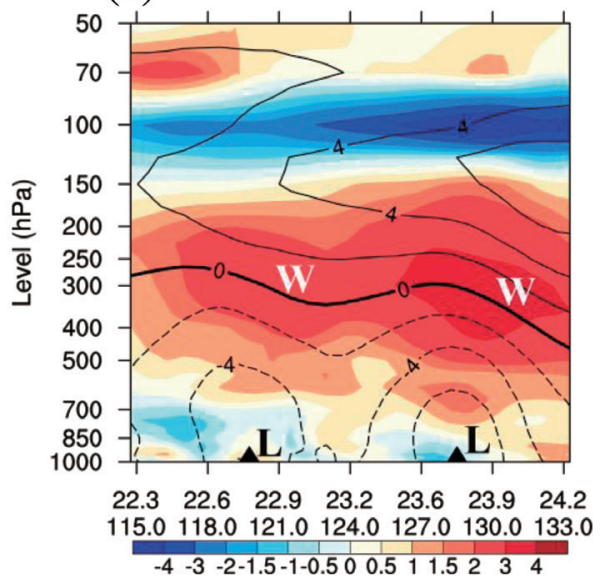

(d)

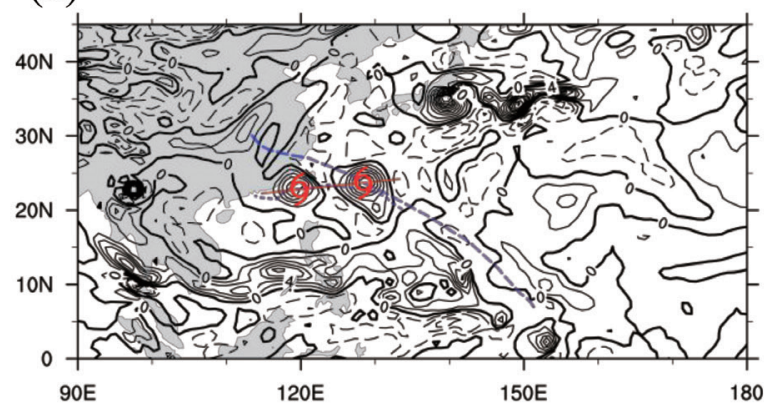

Fig. 13. Same as in Fig. 6 except of (a) 1200 UTC 7 and (b) 0000 UTC, 9 August 2006 for the two TCs of Bopha and Saomai. Panels (c) and (d) are the same as in Fig. 8b except at 1200 UTC, 7 August 2006 and 0000 UTC, 9 August 2006 for the two TCs of Bopha and Saomai at 450 and $500 \mathrm{hPa}$, respectively.

ence on the movement of Fungwong, the reverse was not true. The other two pairs of TCs were also found to have a binary interaction by using complex models, but the TC's intensities and sizes were not objectively determined. The dynamical GBAM model showed that there was not any binary interaction between Goni (2009) and Morakot (2009) before Morakot made landfall on Taiwan Island. The right-turning movement of Morakot near Taiwan Island was influenced by the anomalous subtropical anticyclone in the NW Pacific. The GBAM also showed that Saomai (2002) had a direct influence on the movement of Bopha (2002), but the reverse is not true. The impact of Bopha (2002) on the intensity change of Saomai (2002) and their tracks were accurately simulated by using the Hurricane Weather Research and Forecast System (HWRF) Model (Xu et al. 2013). Many complex models can eventually adequately simulate the intensity evolution and location movement of any TC after many experiments using different initial conditions and physical processes. However, the GBAM, being much simpler, only runs at the optimal level near the maximum center of vorticity anomalies at an initial condition without considering different physical processes.

The intensities of the four studied TC pairs were not similar to each other at the same time during their common lifetime period. The result of GBAM revealed that a stronger TC had a direct influence on the track of a weaker TC, but the reverse was not true. The binary interaction between two vicinity TCs should have equal intensity during their common lifetime period. This situation is not found in this paper, so we will study the binary interaction of two vicinity TCs with similar intensity during their common lifetime period in our following research. This will allow us to obtain a better understanding of the concept of binary interaction proposed and described by Fuji- 
(a)

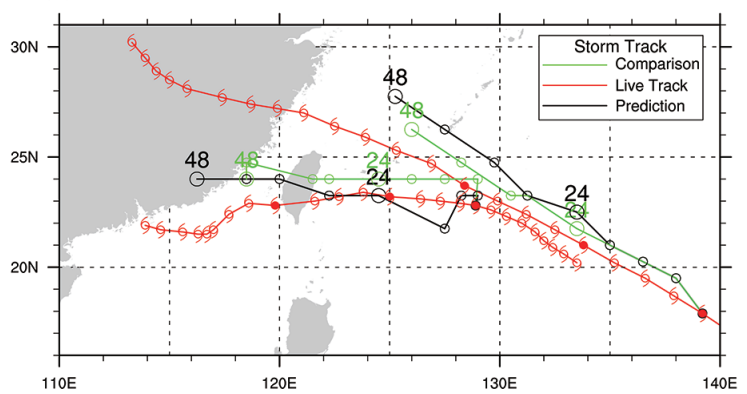

(c)

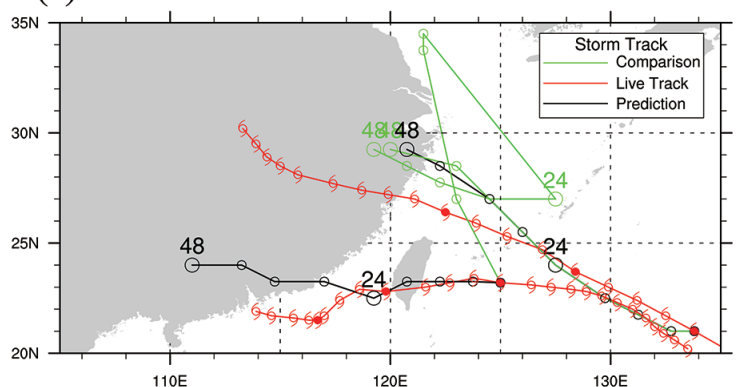

(e)

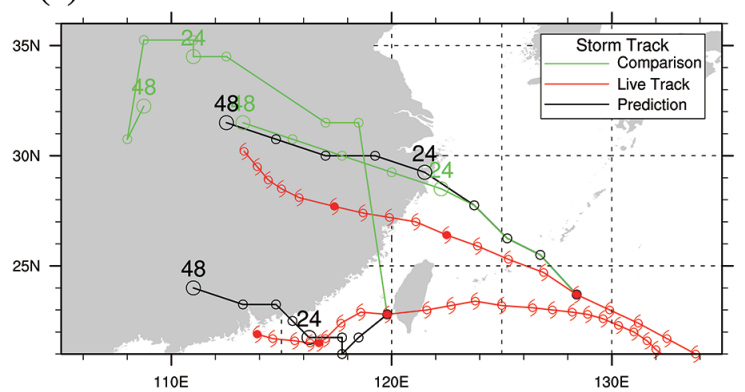

(b)

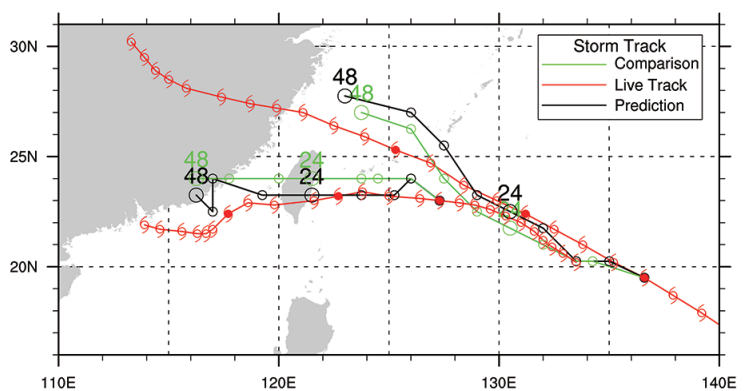

(d)

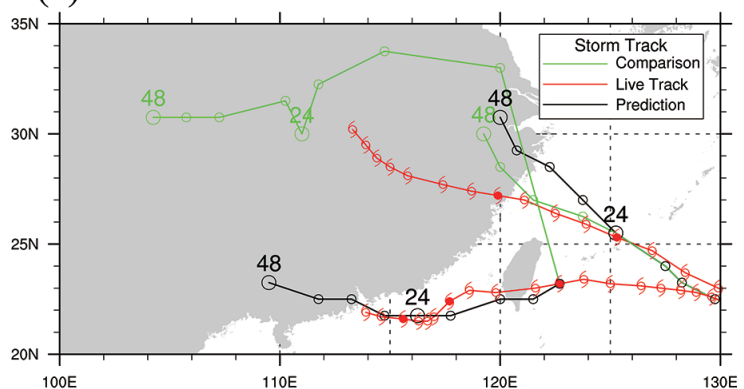

(f)

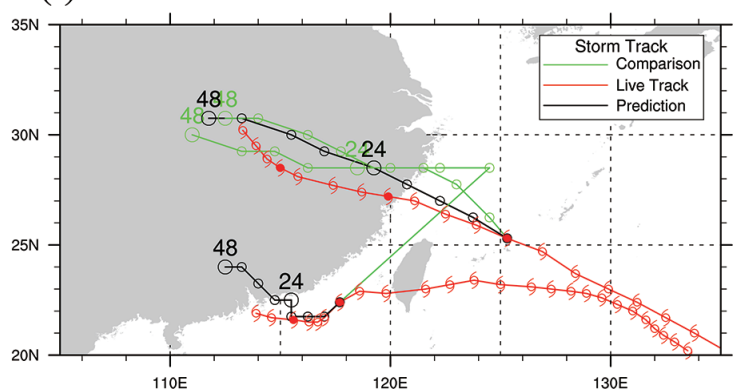

Fig. 14. Same as in Fig. 5, except for the two TCs of Bopha and Saomai initiated respectively at (a) 0000 UTC, 7 August 2006, (b) 1200 UTC, 7 August 2006, (c) 0000 UTC, 8 August 2006, (d) 1200 UTC, 8 August 2006, (e) 0000 UTC, 9 August 2006, and (f) 1200 UTC, 9 August 2006 at 500, 450, 550, 550, 850, and $850 \mathrm{hPa}$, respectively.

whara $(1921,1923,1931)$ in his tank experiments many years ago.

\section{Acknowledgments}

The authors wish to thank two anonymous reviewers and our editors for their valuable comments and suggestions to improve the paper. This work is supported by the National Natural Science Foundation of China (41375073) and the Key Technologies R\&D Program (201306032) and the Global Change and Air-Sea Interaction Program (GASI-03-02-01-02).

\section{References}

Bai, L.-N., L.-M. Ma, Z.-H. Zeng, W. Huang, and D.-L. Wang, 2014: Numerical analysis on the role of tropical storm Namtheum in the unusual tracks of three tropical cyclones in 2010. J. Trop. Meteor, 20, 297-307.

Chan, J. C. L., 2005: The physics of tropical cyclone motion. Annu. Rev. Fluid Mech., 37, 99-128.

Chan, J. C. L., and W. M. Gray, 1982: Tropical cyclone movement and surrounding flow relationship. Mon. Wea. Rev., 110, 1354-1374.

Chen, L.-S., and Y.-H. Ding, 1979: An Introduction to the Western Pacific Typhoons. Science Press, Beijing, 
China, $491 \mathrm{pp}$ (in Chinese).

Chien, F.-C., 2014: A numerical study on the slow translation speed of Typhoon Morakot (2009). SOLA, 10, 190-193.

Chu, J.-H., C. R. Sampson, A. S. Levine, and E. Fukada, 2002: The Joint Typhoon Warning Center tropical cyclone best tracks 1945-2000. Joint Typhoon Warning Center, Pearl Harbor, Hawaii, NRL/ MR/7540-02-1602.

Davis, C., C. Snyder, and A. C. Didlake, 2008: A vortexbased perspective of eastern Pacific tropical cyclone formation. Mon. Wea. Rev., 136, 2461-2477.

Dee, D. P., S. M. Uppala, A. J. Simmons, P. Berrisford, P. Poli, S. Kobayashi, U. Andrae, M. A. Balmaseda, G. Balsamo, P. Bauer, P. Bechtold, A. C. M. Beljaars, L. van de Berg, J. Bidlot, N. Bormann, C. Delsol, R. Dragani, M. Fuentes, A. J. Geer, L. Haimberger, S. B. Healy, H. Hersbach, E. V. Hólm, L. Isaksen, P. Kållberg, M. Köhler, M. Matricardi, A. P. McNally, B. M. Monge-Sanz, J.-J. Morcrette, B.-K. Park, C. Peubey, P. de Rosnay, C. Tavolato, J.-N. Thépaut, and F. Vitart, 2011: The ERA-Interim reanalysis: Configuration and performance of the data assimilation system. Quart. J. Roy. Meteor. Soc., 137, 553-597.

Deng, G., Y.-S. Zhou, and L.-P. Liu, 2010: Use of a new steering flow method to predict tropical cyclone motion. J. Trop. Meteor., 16, 154-159.

Ding, T., and W.-H. Qian, 2012: Statistical characteristics of heat wave precursors in China and model prediction. Diqiu Wuli Xuebao, 55, 1472-1486 (in Chinese).

Dong, K., and C. J. Neumann, 1986: The relationship between tropical cyclone motion and environmental geostrophic flows. Mon. Wea. Rev., 114, 115-122.

Franklin, J. L., 1990: Dropwindsonde observations of the environmental flow of hurricane Josephine (1984): Relationships to vortex motion. Mon. Wea. Rev., 118, 2732-2744.

Fujiwhara, S., 1921: The natual tendency towards symmetry of motion and its application as a principle in meteorology. Quart. J. Roy. Meteor. Soc., 47, 287-293.

Fujiwhara, S., 1923: On the growth and decay of vortical systems. Quart. J. Roy. Meteor. Soc., 49, 75-104.

Fujiwhara, S., 1931: Short note on the behavior of two vortices. Proc. Phys. Math. Soc. Japan, Ser. 3, 13, 106-110.

Galarneau, Jr., T. J., and C. A. Davis, 2013: Diagnosing forecast errors in tropical cyclone motion. Mon. Wea. Rev., 141, 405-430.

George, J. E., and W. M. Gray, 1976: Tropical cyclone motion and surrounding parameter relationships. $J$. Appl. Meteor., 15, 1252-1264.

George, J. E., and W. M. Gray, 1977: Tropical cyclone recurvature and nonrecurvature as related to surrounding wind-height fields. J. Appl. Meteor., 16, 34-42.

Holland, G. J., 1983: Tropical cyclone motion: Environ- mental interaction plus a beta effect. J. Atmos. Sci., 40, 328-342.

Holland, G. J., 1984: Tropical cyclone motion: A comparison of theory and observation. J. Atmos. Sci., 41, $68-75$.

Huang, H.-L., M.-J. Yang, and C.-H. Sui, 2014: Water budget and precipitation efficiency of Typhoon Morakot (2009). J. Atmos. Sci.,71, 112-129.

Huang, J., J. Du, and W.-H. Qian, 2015: A comparison between Generalized Beta-Advection Model and classical Beta-Advection Model in predicting and understanding unusual typhoon tracks in eastern China seas. Wea. Forecasting, 30, 771-792.

Jang, W., and H.-Y. Chun, 2012: The effects of topography on the evolution of Typhoon Saomai (2006) under the influence of tropical stiorm Bopha (2006). Mon. Wea. Rev., 141, 468-489.

Marks, D. G., 1992: The beta and advection model for hurricane track forecasting, NOAA Tech. Memo. NWS NMC-70, 89 pp.

Lee, H. S., T. Yamashita, J. R.-C. Hsu, and F. Ding, 2013: Integrated modeling of the dynamic meteorological and sea surface conditions during the passage of Typhoon Morakot. Dyn. Atmos. Oceans, 59, 1-23.

Liou, Y.-C., T.-C. C. Wang, Y. C. Tsai, Y.-S. Tang, P.-L. Lin, and Y.-A. Lee, 2013: Structure of precipitating systems over Taiwan's complex terrain during Typhoon Morakot (2009) as revealed by weather radar and rain gauge observations. J. Hydrol., 506, 14-25.

Lu, B, and W. H. Qian, 2012: Seasonal lock of rapidly intensifying typhoons over the South China offshore in early fall. Chinese J. Geophys., 55, 1523-1531(in Chinese).

Peng, X., Y. Che, and J. Chang, 2013: A novel approach to improve numerical weather prediction skills by using anomaly integration and historical data. J. Geophy. Res., 118, 8814-8826.

Qian, W.-H., 2012a: Principles of Medium to Extended Range Weather Forecasts. vol. 1-33, Science Press, Beijing, China, 410 pp (in Chinese).

Qian, W.-H., 2012b: Physical decomposition principle of regional-scale atmospheric transient anomaly. Chinese J. Geophys., 55, 1439-1448.

Qian, W.-H., and Z.-J. Zhang, 2012: Precursors to predict low-temperature freezing-rain events in southern China. Chinese J. Geophys., 55, 1501-1512 (in Chinese).

Qian, W.-H., X. Shan, and Y.-F. Zhu, 2012: Capability of regional-scale transient wind anomalies to indicate regional heavy rains. Chinese J. Geophys., 55, 15131522.

Qian, W.-H., J. Li, and X. Shan, 2013: Application of synoptic-scale anomalous winds predicted by medium-range weather forecast models on the regional heavy rainfall in China in 2010. Sci. China. Earth 
Sci., 56, 1059-1070.

Qian, W.-H., X. Shan, H. Liang, J. Huang, and C.-H. Leung, 2014: A generalized beta advection model to improve unusual typhoon track prediction by decomposing total flow into climatic and anomalous flows. $J$. Geophys. Res., 119, 1097-1117.

Roy, C., and R. Kovordanyi, 2012: Tropical cyclone track forecasting techniques-A review. Atmos. Res., 104, 40-69.

Sanders, F., A. L. Adams, N. J. B. Gordon, and W. D. Jensen., 1980: Further development of a barotropic operational model for predicting paths of tropical storms. Mon. Wea. Rev., 108, 642-654.

Simpson, R., 2003: Hurricane!: Coping with Disaster: Progress and Challenges Since Galveston, 1900. American Geophysical Union, 360 pp.

Tsai, Y., C.-S. Chern, S. Jan, and J. Wang, 2013: Numerical study of cold dome variability induced by Typhoon Morakot (2009) off northeastern Taiwan. J. Mar. Res., 71, 109-131.

Velden, C. S., and L. M. Leslie, 1991: The basic relationship between tropical cyclone intensity and the depth of the environmental steering layer in the Australian region. Wea. Forecasting, 6, 244-253.

Wu, X., J. Fei, X. Huang, X. Cheng, and J. Ren, 2011: Statistical classification and characteristics analysis of binary tropical cyclones over the western North Pacific Ocean. J. Trop. Meteor., 17, 335-344.

Wu, X., J. Fei, X. Huang, X. Zhang, X. Cheng, and J. Ren, 2012: A numerical study of the interaction between two simultaneous storms: Goni and Morakot in September 2009. Adv. Atmos. Sci., 29, 561-574.

Wu, C.-C., T.-S. Huang, W.-P. Huang, and K.-H. Chou, 2003: A new look at the binary interaction: Potential vorticity diagnosis of the unusual southward movement of tropical storm Bopha (2000) and its interaction with Supertyphoon Saomai (2000). Mon. Wea. Rev., 131, 1289-1300.

Wu, L., J. Liang, and C.-C. Wu, 2011: Monsoonal influence on Typhoon Morakot (2009). Part I: Observational analysis. J. Atmos. Sci., 68, 2208-2221.

Wu, L., Z. Ni, J. Duan, and H. Zong, 2013: Sudden tropical cyclone track changes over the western North Pacific: A composite study. Mon. Wea. Rev., 141, 2597-2610.

$\mathrm{Xu}, \mathrm{H} ., \mathrm{X}$. Zhang, and X. Xu, 2013: Impact of tropical storm Bopha on the intensity change of super Typhoon Saomai in the 2006 typhoon season. $A d v$. Meteor, 2013, 487010, doi:10.1155/2013/487010.

Yang, C.-C., C.-C. Wu, K.- H. Chou, and C.-Y. Lee, 2008: Binary interaction between typhoons Fengshen (2002) and Fungwong (2002) based on the potential vorticity diagnosis. Mon. Wea. Rev., 136, 4593-4611.

Yu, C.-K., and L.-W. Cheng, 2013: Distribution and mechanisms of orographic precipitation associated with Typhoon Morakot (2009). J. Atmos. Sci., 70, 28942915.

Zheng, Z.-W., Q. Zheng, C.-Y. Lee, and G. Gopalakrishnan, 2014: Transient modulation of Kuroshio upper layer flow by directly impinging Typhoon Morakot in east of Taiwan in 2009. J. Geophy. Res., 119, 4462-4473. 